\title{
Evaluation of M2-like macrophage enrichment after diffuse traumatic brain injury through transient interleukin-4 expression from engineered mesenchymal stromal cells
}

Syed Faaiz Enam', Sajidur Rahman Kader ${ }^{1}$, Nicholas Bodkin', Johnathan G. Lyon', Mark Calhoun ${ }^{1}$, Cesar Azrak', Pooja Munnilal Tiwari ${ }^{2}$, Daryll Vanover ${ }^{2}$, Haichen Wang ${ }^{3}$, Philip J. Santangelo ${ }^{2}$ and Ravi Venkat Bellamkonda ${ }^{{ }^{*}}$

\begin{abstract}
Background: Appropriately modulating inflammation after traumatic brain injury (TBI) may prevent disabilities for the millions of those inflicted annually. In TBI, cellular mediators of inflammation, including macrophages and microglia, possess a range of phenotypes relevant for an immunomodulatory therapeutic approach. It is thought that early phenotypic modulation of these cells will have a cascading healing effect. In fact, an anti-inflammatory, "M2-like" macrophage phenotype after TBI has been associated with neurogenesis, axonal regeneration, and improved white matter integrity (WMI). There already exist clinical trials seeking an M2-like bias through mesenchymal stem/stromal cells (MSCs). However, MSCs do not endogenously synthesize key signals that induce robust M2-like phenotypes such as interleukin-4 (IL-4).
\end{abstract}

Methods: To enrich M2-like macrophages in a clinically relevant manner, we augmented MSCs with synthetic IL-4 mRNA to transiently express IL-4. These IL-4 expressing MSCs (IL-4 MSCs) were characterized for expression and functionality and then delivered in a modified mouse TBI model of closed head injury. Groups were assessed for functional deficits and MR imaging. Brain tissue was analyzed through flow cytometry, multi-plex ELISA, qPCR, histology, and RNA sequencing.

Results: We observed that IL-4 MSCs indeed induce a robust M2-like macrophage phenotype and promote antiinflammatory gene expression after TBI. However, here we demonstrate that acute enrichment of M2-like macrophages did not translate to improved functional or histological outcomes, or improvements in WMI on MR imaging. To further understand whether dysfunctional pathways underlie the lack of therapeutic effect, we report transcriptomic analysis of injured and treated brains. Through this, we discovered that inflammation persists despite acute enrichment of M2-like macrophages in the brain.

(Continued on next page)

\footnotetext{
* Correspondence: ravi@duke.edu

${ }^{1}$ Department of Biomedical Engineering, Duke University, Durham, NC, USA

Full list of author information is available at the end of the article
}

(c) The Author(s). 2020 Open Access This article is licensed under a Creative Commons Attribution 4.0 International License, which permits use, sharing, adaptation, distribution and reproduction in any medium or format, as long as you give appropriate credit to the original author(s) and the source, provide a link to the Creative Commons licence, and indicate if changes were made. The images or other third party material in this article are included in the article's Creative Commons licence, unless indicated otherwise in a credit line to the material. If material is not included in the article's Creative Commons licence and your intended use is not permitted by statutory regulation or exceeds the permitted use, you will need to obtain permission directly from the copyright holder. To view a copy of this licence, visit http://creativecommons.org/licenses/by/4.0/ The Creative Commons Public Domain Dedication waiver (http://creativecommons.org/publicdomain/zero/1.0/) applies to the data made available in this article, unless otherwise stated in a credit line to the data. 


\begin{abstract}
(Continued from previous page)
Conclusion: The results demonstrate that MSCs can be engineered to induce a stronger M2-like macrophage response in vivo. However, they also suggest that acute enrichment of only M2-like macrophages after diffuse TBI cannot orchestrate neurogenesis, axonal regeneration, or improve WMI. Here, we also discuss our modified TBI model and methods to assess severity, behavioral studies, and propose that IL-4 expressing MSCs may also have relevance in other cavitary diseases or in improving biomaterial integration into tissues.
\end{abstract}

Keywords: Interleukin-4, IL-4, mRNA, Stem cell, MSC, Macrophage, M2, Traumatic brain injury, TBI, RNA-seq

\section{Background}

Traumatic brain injury (TBI) places a calamitous toll on individuals and the health system. TBI accounts for 138 deaths every day and 2.5 million emergency room visits, hospitalizations, or deaths annually [1]. It makes up one third of all "Unintentional Injuries," the leading cause of mortality between the ages 1 and 44. This has economic ramifications on the order of $\$ 60-80$ billion per year [2, $3]$. TBI is divided into mild, moderate, and severe categories. While mild TBI is typically thought to result in a transient loss of function, studies are elucidating persisting complications [4]. Severe TBI results in permanent morbidity or mortality in all patients [5].

However, the permanent morbidity and mortality are seldom due to the primary insult and instead result from numerous injury-associated sequelae. These include inflammation, excitotoxicity, accumulation of reactive oxygen species, apoptosis of injured cells, ischemia, edema, and blood-brain barrier (BBB) disruption [5]. Soon after TBI, damaged cells release distress signals, "alarmins," consisting of intracellular components and various cytokines [6]. Some alarmins reach the bloodstream to recruit neutrophils and monocytes that can morph into macrophages in the parenchyma. Other alarmins activate microglia to clear debris and activate astrocytes to reestablish barriers. At first, these mechanisms are neuroprotective but over weeks or months they become maladaptive. In mammals, the microglia and macrophages stay persistently skewed toward proinflammatory, M1-like phenotypes [7-11]. They selfamplify by releasing more inflammatory cytokines and can remain at injury site for weeks and months [7]. The M1-like response in lower-order animals, like zebrafish, is abrogated by a robust "anti-inflammatory" M2-like response within a week, enabling them to regenerate a transected spinal cord [12, 13]. Cohorts of M1 and M2 macrophages were first identified in vitro and in other injury conditions [14], but the phenotypes can exist concurrently after TBI [15]. Additionally, it is now well understood that macrophages in vivo encompass a phenotype across an M1-M2 spectrum. Thus, we use the term "M2-like," especially for in vivo macrophages, to suggest a phenotype similar to M2 macrophages previously studied in vitro, as we [16] and others have previously $[17,18]$. These M2-like macrophages are, at least indirectly, associated with improved biological and functional recovery in the mammalian peripheral and CNS after injury [10, 19-23]. They are involved in angiogenesis, neurogenesis, axonal regeneration, and improved white matter integrity [24, 25]. Therapies that enhance an M2-like phenotype after TBI, often improve molecular markers, edema, white matter, and functional outcomes [26-41]. However, the peak M2-like response 5 days after injury is still overwhelmed by M1-like macrophages and the desirable response recedes by day $7[8$, 9]. Shifting the balance toward endogenous, "anti-inflammatory," M2-like macrophages is thus a viable goal for TBI but requires a clinically relevant strategy.

Stem cells are a promising means to promote an antiinflammatory response after TBI and to possibly modulate macrophage phenotypes [42-47]. In fact, a handful of different bone-marrow derived stem cells (BMSCs) are in at least 8 clinical trials $[42,43]$. These cells include multi-potent adult progenitor cells (MAPCs) and mesenchymal stem/stromal cells (MSCs). Phase I trials have determined that harvesting BMSCs from a patient and delivering them is logistically feasible and safe [43]. Encouragingly, at least one phase I study has reported decreased neural tissue loss and an improvement in a few clinical outcomes at 6 months. Phase II trials are underway in which they compare doses of BMSCs to reduce neuroinflammation after TBI in adults and children. For TBI, one of the more frequently studied stem cells is the MSC [42]. Like many other stem cells, they exert their effects not through differentiation but via secretion of cytokines and growth factors, their "secretome." However, while many pre-clinical studies demonstrate their ability to temper inflammation, skepticism toward MSCs persists [43].

Part of the skepticism toward MSC therapy is in the absence of certain growth factors and cytokines in the MSC secretome [43, 48-52]. One absent cytokine, that also promotes an M2 macrophage phenotype, is interleukin-4 (IL-4) $[49,53,54]$. IL-4 is a small, $16-\mathrm{kDa}$ protein that is classically secreted by and activates Th2 helper cells. However, when it binds to its cognate receptor IL-4R $\alpha$, it initiates STAT6-based transcription and expression of M2 phenotypic proteins on 
macrophages. In vivo, IL-4 promotes M2-like macrophage phenotypes after stroke $[55,56]$, spinal cord injury (SCI) [57-59], and peripheral nerve injury (PNI) [22, 60] at doses ranging from 250 to $500 \mathrm{ng}$. IL-4 stimulates astrocytes to secrete growth factors and promotes microglia to express M2 phenotypic markers as well [61]. Unfortunately, IL-4 levels do not increase in humans or mice after TBI $[62,63]$. In rats, however, there is a modest IL-4 elevation in the first $24 \mathrm{~h}$, but this subsides within 3 days [64]. Delivery of IL-4 after TBI remains unpublished although it is actively being explored (1I01BX003377-01) [65]. Altogether, this suggests that augmenting the MSC secretome with IL-4 could bias macrophages after TBI to a reparative, M2-like, phenotype.

MSCs have previously been genetically altered to express desirable proteins. For rodent models of CNS injury, MSCs have been modified to overexpress bonederived neurotrophic factor (BDNF) [66], superoxide dismutase 2 (SOD2) [67], interleukin-13 (IL-13) [68-70], or interleukin-10 (IL-10) [50, 52]. While most of these modifications have improved molecular parameters in each study, improved functional outcomes are only occasionally observed. BDNF overexpression mildly improved outcomes in one functional SCI assessment [66]. SOD2 overexpression demonstrated improved motor coordination at one time point tested after focal TBI [67]. IL-13 overexpression promotes an M2-like phenotype, but it has only been observed to improve some functional assessments in an SCI model and failed to rescue function in stroke and epilepsy models [68-70]. IL-10 overexpression from MSCs did not reduce TBI lesion volume or improve most functional assessments [50, 52]. The one functional test that improved (foot faults) and a decreased histological presence of reactive astrocytes was not significantly different from wild-type MSCs.

Genetic modification of MSCs to express IL-4 can be achieved via plasmid DNA transfection, viral transduction of DNA, or through synthetic mRNA. MSCs have previously been transduced to express IL-4 and consequently promoted a Th2 response in a model of autoimmune encephalitis [54]. Modification of MSCs in this manner requires DNA that encodes IL-4 to enter the nucleus and then induces prolonged or permanent expression of IL-4. Persistent IL-4 expression, although initially therapeutic, is undesirable as it promotes a chronic M2-like macrophage presence which results in fibrosis [34, 71]. This persistent overexpression can be advantageous outside the nervous system, implanted MSCs over-expressing IL-4 increase bone mineral density [53]. However, in the CNS, fibrosis limits regeneration [72]. Viral transduction also comes with the risk of undesired genomic integration and activation of oncogenes. In contrast, synthetic mRNA only needs to reach ribosomes in the cytosol, thus enabling rapid expression and a short persistence of 1-4 days [50]. This approach could counter an M1-like response faster and allow the injured site to return to homeostasis. Lower-order animals that regenerate their CNS show a similar dynamic: proinflammatory followed by anti-inflammatory peaks that all subside to homeostasis within a couple weeks [12, 13].

Delivering recombinant IL-4 alongside MSCs is another strategy though it can also have drawbacks. As mentioned, IL-4 alone has been delivered after stroke, SCI, and PNI. This may have rescued functional deficits in IL-4 knockout mice suffering stroke [55]. Reports in SCI are conflicting where one study showed moderate functional improvement [57], while the other demonstrated no significant functional improvement [59]. Apart for the difficulty in correctly and robustly conducting behavioral studies, this ambiguity could be because recombinant protein delivery has yet to fully address the challenges of short half-lives [73, 74], suboptimal efficacy [75], and immunogenicity [76, 77]. Cytokines possess short half-lives due to rapid circulation and clearance in vivo and this is exacerbated by increased interstitial flow after TBI. In mouse models, IL-4 half-life can be less than $20 \mathrm{~min}$ [54, 78]. To counter this, multi-dosing or sustained release strategies need to be employed. A lack of post-translational modifications (PTMs) and the presence of microstructural abnormalities can further worsen half-life, reduce efficacy, and increase immunogenicity. These challenges can be addressed by inducing harvested MSCs to express IL-4 via nucleic acids as unsullied IL-4 can be synthesized with endogenous PTMs.

In the present study, we transfect MSCs with synthetic IL-4 mRNA to transiently induce IL-4 expression. We hypothesize that these modified MSCs will promote M2like macrophage-mediated healing in a modified closed head injury (CHI) mouse model of TBI. Through our model, we investigate in vivo macrophage polarization, examine behavioral outcomes, scrutinize cytokine and gene expression, probe histological alterations in tissue and white matter tracts, and explore transcriptomic dysregulation.

\section{Materials and methods}

\section{In vitro transcribed mRNA (IL-4, luciferase, GFP)}

All synthetic mRNA were in vitro transcribed in the Santangelo Lab (Georgia Institute of Technology, USA) similarly to past research [79].

\section{Cells (MSCs, macrophages)}

Mesenchymal stem/stromal cells (MSCs) were purchased at passage 6 from Cyagen Biosciences (USA) (Lot\# 170221|31). These MSCs are primary cells 
harvested by the vendor from $\mathrm{C} 57 \mathrm{BL} / 6 \mathrm{~N}$ mice. All in vivo experiments used the same strain of mice. According to the vendor, the MSCs express CD29, CD44, and Sca-1 and do not express CD31 and CD45. We verified this expression in two late passages (S. Fig. 1). The MSCs were grown in Mouse Mesenchymal Growth Media (Cyagen Biosciences, USA) with 10\% FBS (Lot\# T161102G002) and frozen at passage 7 and stored in the vapor phase of liquid nitrogen. For every in vivo experiment, the cells were thawed, and grown in media for 34 days before experiments. To passage or plate cells, the cells were washed twice with PBS (Corning, USA). Then, 0.25\% Trypsin (Corning, USA) was added, with enough volume to cover the surface area, and gently rocked for $90 \mathrm{~s}$. The Trypsin was suctioned off, and the cells were left in a $37^{\circ} \mathrm{C}$ incubator for $1 \mathrm{~min}$. Finally, the cells were harvested by adding fresh, pre-warmed, and $\mathrm{pH}-$ equilibrated media to the cells.

For macrophage polarization experiments, J774A.1 macrophages were obtained from the Cell Culture Facility at Duke University (thawed from an unknown passage number). These were grown in DMEM-High Glucose (Gibco, USA) supplemented with 10\% FBS. Cells were passaged by manual dissociation with a cell scraper.

\section{Transfection}

Viromer Red (Lipocalyx, USA), jetPEI (Polyplus Transfection, USA), and Lipofectamine Messenger Max (ThermoFisher, USA) were used to transfect MSCs with GFP mRNA following manufacturer protocol. All subsequent MSC transfections of synthetic IL-4 or Luciferase mRNA used Viromer Red, following its protocol. Prior to every transfection, a cell culture cabinet, pipettes, and other materials were decontaminated with $70 \%$ ethanol and RNAse Away (ThermoFisher, USA) and left to dry.

\section{In vitro flow cytometry}

For MSC cell-surface characterization, the following antibodies were used: APC-Vio770 CD29 (Miltenyi Biotec; clone, HM $\beta 1-1$ ), VioBright FITC CD44 (Miltenyi Biotec, USA; clone, REA665), PerCP Sca-1 (Biolegend, USA; clone, D7), PE CD31 (Miltenyi Biotec, USA; clone, 390), PE CD45 (Biolegend, USA; clone, 30-F11), and PEVio770 CD105 (Miltenyi Biotec, USA; clone, MJ7/19). For analysis of in vitro macrophages, we first blocked Fc receptors with CD16/32 Fc $\gamma$ RIII/Fc $\gamma$ RII (BD Bioscience, USA; clone, 2.4G2) and CD16.2 FcrRIV (Biolegend, USA; clone, 9E9) and then stained with PE CD45 (Biolegend, USA; clone, 30-F11), Alexa Fluor 647 CD206 (BioLegend, USA; clone, C068C2), and PE CD163 (ThermoFisher, USA; clone, TNKUPJ). A Novocyte 2060 (ACEA Biosciences, USA) was used to measure mean fluorescence intensity of GFP from transfected MSCs,
MSC cell-surface markers, and macrophage markers. Data was analyzed in FlowJo software (BD Biosciences, USA).

\section{In vitro assays: viability, ELISA, and imaging}

Cell viability was assessed with either a Live/Dead Viability/Cytotoxicity kit (ThermoFisher, USA) and DMi8 LiveCell Microscope (Leica Biosystems, USA) or 0.4\% Trypan Blue (ThermoFisher, USA) exclusion with automated cell counting using a calibrated Countess II (ThermoFisher, USA). For protein quantification of conditioned media, an IL-4 colorimetric ELISA was performed (R\&D DuoSet; R\&D Systems, USA) with appropriate ancillary kits. Optical density was obtained via a 96-well plate reader (SpectraMax i3x, Molecular Devices, USA). In some experiments, prior to media collection, cells were imaged under brightfield in the DMi8 LiveCell Microscope and the images were analyzed with a custom Image J script to quantify cell coverage of the well in each well as an estimator of cell confluency/ count.

\section{Closed head injury}

All in vivo experiments were conducted according to protocols approved by the Duke Institute for Animal Care and Use Committee (IACUC). Male C57BL/6N mice were purchased from Charles River Laboratories, USA, and left to acclimate for 1-2 weeks in Duke University animal housing. Procedures began when the mice were between 8 and 9 weeks of age.

Closed head injury (CHI) experiments were modified from Webster et al. to develop a model that is easily reproducible but also induces behavioral deficits in motor coordination [80]. Mice were induced under 5\% isoflurane vapor anesthesia and maintained between 1 and 3\% anesthesia. Hair was trimmed from the scalp and ointment was applied on the eyes (Paralube; Dechra, USA). The scalp was sterilized with three $70 \%$ ethanol and chlorhexidine swabs each. A mid-line cut into the scalp was made with micro-scissors, the cranium was exposed, and the bone surface cleaned with a cotton-tip swab. The mouse was then transferred onto a custom heated bed in a rat stereotactic apparatus (51600; Stoelting, USA) with mouse gas adaptor (10030-386; VWR, USA), and mouse non-rupture ear bars (922; Kopf Instruments, USA) connected to a separate anesthesia system maintaining 1-3\% anesthesia. A balloon connected through tubing to a $20-\mathrm{mL}$ syringe was also placed under the head of the mouse to prevent skull fracture [80]. A 5$\mathrm{mm}$ impact probe connected to an electromagnetic impact system (Impact One; Leica Biosystems, USA) was extended and positioned $-1.5 \mathrm{~mm}$ AP from bregma and zeroed to the surface. At this point, anesthesia was reduced to $0.5 \%$, oxygen flow increased to $2 \mathrm{~L} / \mathrm{min}$, and 
the impactor retracted and then lowered $1.5 \mathrm{~mm}$ DV. When breathing became faster, a toe-pinch was assessed to ensure no pain sensation, and the impact was hit at 6 $\mathrm{m} / \mathrm{s}$. If the toe-pinch induced a reflex, injury was not performed, anesthesia was immediately increased, and the steps were repeated. Controlling anesthesia levels carefully is critical to survival; if the anesthesia is too deep prior to impact, the mouse may not return to spontaneous breathing after the impact. Immediately after impact, the oxygen flow was increased to $4 \mathrm{~L} / \mathrm{min}$ and the mouse was placed on its right side for chest compressions carried out with an index finger and thumb. When the mouse began to breathe spontaneously, the scalp incision was stapled (Reflex 7-mm wound clips, Roboz Surgical, USA), 3 drops of $0.5 \%$ bupivicaine were added to the site, and the mouse placed supine in an empty cage. Time for the mouse to right itself into a prone position was recorded. Righting was considered when all four paws were on the cage floor. If there was a fracture, brain hernia, or excessive bleeding after the impact, the mouse was immediately put back under $5 \%$ anesthesia and then euthanized. If righting time was > $10 \mathrm{~min}$, it was considered an injury [81]. Neurologic Severity Score (NSS) was assessed in two experiments as per its protocol [82]. With two scientists working on mouse preparation and injury separately, injuries can be 5-10 min apart. Sham procedures consisted of all steps except for the impact.

\section{Preparation and delivery of MSCs}

Based on transfection and expression data (Fig. 3a), an mRNA incubation time of $10 \mathrm{~h}$ was chosen. On the day prior to injections, cultured MSCs were plated in the afternoon in 6-well plates at 300,000 cells per well. After at least $6 \mathrm{~h}, \mathrm{MSCs}$ were transfected with $2 \mu \mathrm{g}$ mRNA complexed with Viromer Red according to manufacturer protocol. Ten hours after transfection, the MSCs were harvested with two 1-mL collections of media per well. All similar wells were grouped in $15 \mathrm{~mL}$ tubes and spun down at $300 \times g$ for $3 \mathrm{~min}$. The media was suctioned, and the cells were resuspended in $1 \mathrm{~mL}$ of PBS. The cells were counted via a cell counter (Countess II; ThermoFisher, USA) that was previously calibrated to manual cell counting. The cells were then spun again and resuspended to make a 30 million cells $/ \mathrm{mL}$ mixture in PBS. Aliquots of $10 \mu \mathrm{L}$ were made and kept on ice until injection.

To deliver MSCs, intrahippocampal injections were conducted 2 or 5 days after injury. As previously, the mice were induced with $5 \%$ isoflurane and maintained between 1 and 3\% anesthesia. The eyes were protected with ointment, the surgical site was cleaned with ethanol and chlorhexidine, staples were removed with a staple remover, the old incision was opened with micro- scissors, and the skull surface was cleaned with a cottontip swab. The mouse was moved to the rat stereotactic apparatus with a different mouse gas adaptor (923-B; Kopf Instruments, USA). A craniotomy was performed with a 0.6-mm drill-bit (Roboz Surgical, USA) attached to a handheld drill (Stoelting, USA) at $-1.5 \mathrm{~mm}$ AP, and $-1 \mathrm{~mm}$ ML (left) drilling $0.4-0.6 \mathrm{~mm}$ deep. Cells were then mixed and picked up by a $5-\mu \mathrm{L}$ syringe $(75 \mathrm{RN}$; Hamilton, USA) with a 26-G needle (1-in., point style 4, $30^{\circ}$; Hamilton, USA). This needle was chosen as it had the closest inner-diameter to the syringe. The syringe was attached to the stereotactic apparatus and inserted to a depth of $2 \mathrm{~mm}$ DV from the outer surface and then retracted $0.5 \mathrm{~mm}$ for a final injection location of $1.5 \mathrm{~mm}$ DV (left hippocampus). For ventricular injections, the coordinates were $-0.5 \mathrm{AP},-1 \mathrm{ML}, 2.25 \mathrm{DV}$. Cells were injected at a rate of $0.5 \mu \mathrm{L} / \mathrm{min}$ in a volume of $5 \mu \mathrm{L}$.

\section{Behavioral experiments (rotarod, Morris water maze)}

To assess motor coordination and function, mice were tested on an accelerating rotarod apparatus (Med Associates Inc., USA). On the day prior to $\mathrm{CHI}$, the mice first underwent a training trial on the rotarod where they were put back on as they fell until $400 \mathrm{~s}$ passed. The rotarod accelerated from 4 to 40 rotations per minute. After that, three recording trials were run with at least 15 min between each trial. Trials were stopped (and recorded) if the mouse stayed on for $400 \mathrm{~s}$ or if it held onto the rod without running for two spins. The time on the rotarod was recorded.

To assess spatial memory function, a Morris water maze was set up with a large round pool, four images on the walls as cues, a glass platform hidden under the surface of the water, and a camera and computer set up with tracking software (ANY-maze; Stoelting, USA). Mice were introduced into the pool starting at the edge of the pool and time to find the hidden platform was recorded. Four trials were conducted each day, each starting at one pole of the pool (North, South, East, or West).

\section{Perfusion/euthanasia}

All mice were sacrificed under 5\% isoflurane anesthesia and cardiac perfusion. Perfusate consisted of Hank's Balanced Salt Solution (Corning, USA) with Heparin (10, 000 units/L; ThermoFisher, USA) kept on ice. After testing for a reaction to toe-pinch, the sternum was lifted, and the abdomen and ribs cut with scissors. The diaphragm was dissected away, and the heart was exposed and freed from connective tissue. The left ventricle of the heart was pierced with a $26-\mathrm{G} 3 / 8$ " needle (BD Biosciences, USA) and the mouse perfused at a rate of 8 $\mathrm{mL} / \mathrm{min}$ for at least $2.5 \mathrm{~min}$ with a motorized pump (Cole-Parmer, USA). For immunohistochemistry, this 
was followed by cold $4 \%$ formaldehyde in HBSS. The mouse was then decapitated, and the brain harvested. For protein and gene expression studies, the brain was further dissected to isolate the injection region (3- $\mathrm{mm}$ width per hemisphere), stored in $5 \mathrm{~mL}$ conical tubes, frozen in liquid nitrogen, and stored in a $-80^{\circ} \mathrm{C}$ freezer until processed.

\section{Ex vivo flow cytometry}

To study macrophage polarization, leukocytes were isolated from freshly harvested brain tissue following previously published protocol [83]. The protocol was slightly modified and is included in the supplementary information. As previously, Fc receptors were blocked with antibodies against CD16/32 Fc $\gamma$ RIII/Fc $\gamma$ RII (BD Bioscience, USA; clone, 2.4G2) and CD16.2 FcyRIV (Biolegend, USA; clone, 9E9) and then stained with PE-Cy7 CD45 (BioLegend, USA; clone, I3/2.3), PE F4/80 (BioLegend, USA; clone, BM8), Alexa Fluor 488 CD86 (BioLegend, USA; clone, GL-1), and Alexa Fluor 647 CD206 (BioLegend, USA; clone, C068C2). Macrophages were defined as CD45 ${ }^{\text {high }}, \mathrm{F} 4 / 80^{+}$. Cells were run in a Novocyte 2060 (ACEA Biosciences, USA) at a rate of $14 \mu \mathrm{L} / \mathrm{min}$.

\section{Ex vivo protein quantification}

Frozen brain tissue samples were homogenized in their tubes with a TissueRuptor II (6 speed, $30 \mathrm{~s}$; Qiagen, USA) and disposable probes (Qiagen, USA) on ice with Halt Protease Inhibitor (ThermoFisher, USA) in N-PER reagent (ThermoFisher, USA). Homogenates were spun down thrice. The first spin in the $5 \mathrm{~mL}$ tubes was at $1000 \times g$ for $10 \mathrm{~min}$ at $4{ }^{\circ} \mathrm{C}$. The supernatant was transferred into $2 \mathrm{~mL}$ tubes. The second and third spins were at $12,000 \times g$ for $15 \mathrm{~min}$ and $15,000 \times g$ for $20 \mathrm{~min}$, respectively, both at $4{ }^{\circ} \mathrm{C}$, with a transfer to new tubes after each spin. The protein samples were then processed with an 8-plex bead-based assay (LEGENDplex Mouse Th1/Th2 Panel; BioLegend, USA) according to manufacturer protocol. Samples were run on a Novocyte 2060 (ACEA Biosciences, USA). Quantification was normalized against a total protein bicinchoninic acid (BCA) assay (ThermoFisher, USA) of each protein sample.

\section{Gene expression and RNA sequencing}

The workspace was first cleaned and sprayed with RNAse Away (ThermoFisher, USA). Frozen brain tissue samples were processed with an RNeasy Lipid Tissue Kit (Qiagen, USA) to extract RNA. The tissue samples were homogenized with a TissueRuptor II (6 speed, $30 \mathrm{~s}$ ) and disposable probes. RNA integrity was assessed on an Agilent Bioanalyzer (Agilent, USA) with the assistance of the Duke Microbiome Shared Resource. Complementary DNA (cDNA) was synthesized with SuperScript IV VILO (ThermoFisher, USA), separated into aliquots and stored at $-80^{\circ} \mathrm{C}$. Genes were studied with TaqMan Gene Expression Assays (ThermoFisher, USA) in 384well plates. The TaqMan GEAs that are published here include il4 (Mm00445259_m1), il10 (Mm01288386_m1), il1b (Mm00434228_m1), il1rn (Mm00446186_m1), mrc1 (Mm01329362_m1), il6ra (Mm01211445_m1), cxcr4 (Mm01996749_s1), erbb2 (Mm00658541_m1), bdnf (Mm04230607_s1), ngf (Mm00443039_m1), ntf3 (Mm00435413_s1), neurog2 (Mm00437603_g1), notch1 (Mm00627185_m1), cspg4 (Mm00507257_m1), sox2 (Mm03053810_s1), bmp2 (Mm01340178_m1), s100b (Mm00485897_m1), artn (Mm00507845_m1), tgfb1 (Mm01178820_m1) ptn (Mm01132688_m1), casp7 (Mm00432322_m1), nrcam (Mm00663607_m1), ngfr (Mm00446296_m1), il10ra (Mm00434151_m1), cd163 (Mm00474091_m1), and ccl2 (Mm00441242_m1). The samples were run on a 7900HT Real-Time PCR system (Applied Biosystems, USA). Data was analyzed using Applied Biosystems Analysis Software available on the ThermoFisher Cloud Connect Utility (https://apps. thermofisher.com/apps/spa). Gene/sample combinations that had high variation among technical replicates were flagged and rejected. To normalize the amount of mRNA per sample, global normalization was selected instead of using housekeeping genes (due to observed variability) [84], and Sham was kept as the reference ddCT values.

For RNA sequencing, total RNA was sequenced through Illumina NovaSeq 6000 (50 bp, paired-ends). The raw data was quality-checked, adaptors were trimmed with cutadapt (v2.4), and then pseudo-aligned using Kallisto (v0.46.0) against protein-coding GENCODE transcripts (vM23) [85], with differential expression assessment via Sleuth (v0.30.0) in $\mathrm{R}$ (v3.6.1) [86, 87]. Data were consolidated and organized using Python (v3.6.5, w/ pandas 0.24.2, numpy 1.16.4) and then run through an over-representation analysis (g:Profiler, rev 2fcb244) and Gene Set Enrichment Analysis (GSEA, v4.0.3) $[88,89]$. One sample was excluded due to anomalously different transcript counts $899-5$, a PBS.3WK replicate). Raw transcriptomic data is accessible via the NIH Gene Expression Omnibus (GSE144193) and analyzed data are included in the supplementary materials.

\section{Immunohistochemistry}

To study histological changes after intervention, 4\% formaldehyde-fixed brains were embedded in optimal cutting temperature compound (Tissue-Tek; VWR, USA) and frozen in a bath of 4-methylbutane chilled in liquid nitrogen. The brains were sectioned in $10-\mu \mathrm{m}$ slices and collected on Superfrost Plus slides (ThermoFisher, USA) with a hydrophobic barrier drawn (ImmEdge Hydrophobic Barrier PAP Pen; Vector Laboratories, USA). Slides were kept at $-20^{\circ} \mathrm{C}$ until ready to be stained. Primary antibodies included chicken anti- 
mouse GFAP (abcam, USA), rabbit anti-mouse Iba1 (abcam, USA), and rat anti-mouse CD68 (abcam, USA). Secondaries included DyLight 488 goat anti-chicken IgY (abcam, USA), Alexa Fluor 594 goat anti-rat IgG, and Alexa Fluor 680 goat anti-rabbit IgG (abcam, USA). Cell nuclei were counterstained with DAPI (Sigma-Aldrich, USA). Coverslips were applied after drops of Fluoromount-G (SouthernBiotech, USA) were added. One group of slides was stained with a Fluoro-jade $C$ kit (Biosensis, USA). Coverslips were applied after drops of DPX Mounting Media (Sigma-Aldrich, USA) were added. All staining procedures were conducted at the same time for each group. Imaging was done in a DMi8 microscope (Leica Biosystems, USA). All images were acquired in the same time period per stain. Images were analyzed with a custom semi-automated script on ImageJ (S. File 1) with regions of interest drawn for hippocampi, cortices, and corpus callosum.

\section{MRI}

Diffusion-weighted magnetic resonance imaging was conducted with the Duke Center for In Vivo Microscopy (CIVM). MR images were acquired on a 7.0-T horizontal bore magnet with Resonance Research gradients providing $\sim 650 \mathrm{mT} / \mathrm{m}$ maximum gradient. The system is controlled by an Agilent Direct Drive console. Specimens were mounted in a 12 -mm-diameter single-sheet solenoid radiofrequency coil. Three-dimensional (3D) diffusionweighted images were acquired with a Stejskal Tanner rf refocused spin echo sequences with TR/TE of 100/21.15 $\mathrm{ms}$ and $b$ values of $4000 \mathrm{~s} / \mathrm{mm}^{2}$. Compressed sensing was used with an acceleration of $8 \times$ to reduce the acquisition time [90]. The result is a 4D image array with isotropic spatial resolution of $45 \mu \mathrm{m}$ (voxel volume of $91 \mathrm{pL}$ ).

The 4 D array $(256 \times 256 \times 420 \times 7)$ was passed to a post processing pipeline that registered each of the diffusion-weighted 3D volumes to the baseline to correct for eddy current distortion. The registered 4D array was passed to DSI Studio which generated scalar images (AD, RD, FA, ClrFA) using the DTI model.

A 3D label set was registered onto each 4D volume in that volume's native reference frame, i.e., the reference frame in which the data were acquired. Waxholm Space (WHS) [91] an isotropic label set for the mouse brain was expanded by Calabrese et al. was extended with an additional 18 regions of interest yielding 166 regions of interest on each half of the brain [92].

\section{Statistics and graphing}

All statistics were performed in Prism V8.2 (GraphPad, USA). Where appropriate, Student's $t$ test, one-way ANOVAs, repeated measures ANOVAs, and two-way ANOVAs were carried out. All data are presented as means \pm standard deviation. Asterisks are denoted in graphs to represent significant $p$ values. If the asterisk(s) is above a flat horizontal bar, it refers to the significance between the two groups at the end of the bars. If the asterisk(s) is above a bracket, it refers to all comparisons within the bracket. If the asterisk(s) is above a group, it means the group was significantly different from all other groups. In this third situation, the highest $p$ value between comparisons is recorded. Graphs were made in Prism V8.2 and converted into figures in Photoshop and Illustrator (Adobe, USA).

\section{Results}

Transfection with Viromer Red and synthetic IL-4 mRNA generates IL-4 expression

As MSCs do not endogenously produce IL-4, introduction of IL-4 genetic information is required. To reliably introduce synthetic IL-4 mRNA into MSCs, transfection agents exist that enable the mRNA to reach the cytosol without degradation. Here, we first obtained MSCs (Cyagen; harvested from C57BL/6N mice; later in vivo studies are conducted in this mouse strain) that expressed putative MSC markers [93], although there was an absence of CD105. The cells retained this marker expression at passages 9 and 12 as well (S. Fig. 1 ), but most experiments were conducted between passages 7 and 9. Next, synthetic mRNA encoding Green Fluorescent Protein (GFP) was combined with one of three transfection agents (jetPEI, Lipofectamine Messenger Max, or Viromer Red) and incubated for $24 \mathrm{~h}$ with MSCs following manufacturer protocol for each agent at $0.5 \times$ and $1 \times$ their recommended dose. The amount of synthetic mRNA ( 250 or $500 \mathrm{ng}$ ) was equivalent between the transfection agents. Of the three, Viromer Red induced the greatest mean fluorescence intensity (MFI) in a flow cytometer and was thus chosen for future transfections (Fig. 1a). Next, we tested Viromer Red toxicity by complexing it with synthetic IL-4 mRNA and incubating four doses with MSCs for $24 \mathrm{~h}$. Regardless of the dose MSC viability, assessed via a live/dead cell assay, remained greater than 95\% (Fig. 1b). Simultaneously, IL-4 ELISA confirmed that at least $150 \mathrm{ng}$ of IL-4 was expressed into the media (1 mL) (Fig. 1c). Without transfection of synthetic IL-4 mRNA, MSCs did not endogenously express IL-4 (Fig. 1d). In another study, we characterized transfection-induced expression over a 48-h period and observed that IL-4 synthesis begins within the first $2-4 \mathrm{~h}$ and the majority is synthesized within the first $24 \mathrm{~h}$ (Fig. 1e). These studies confirm that MSCs do not endogenously express IL-4, and transfection with Viromer Red and synthetic IL-4 mRNA can generate IL- 4 expression for at least $24 \mathrm{~h}$ with acceptably low toxicity.

\section{Expressed IL-4 induces an M2-like phenotype in vitro}

Next, we set out to determine whether IL-4 secreted from transfected MSCs was functional. IL-4 is a potent 


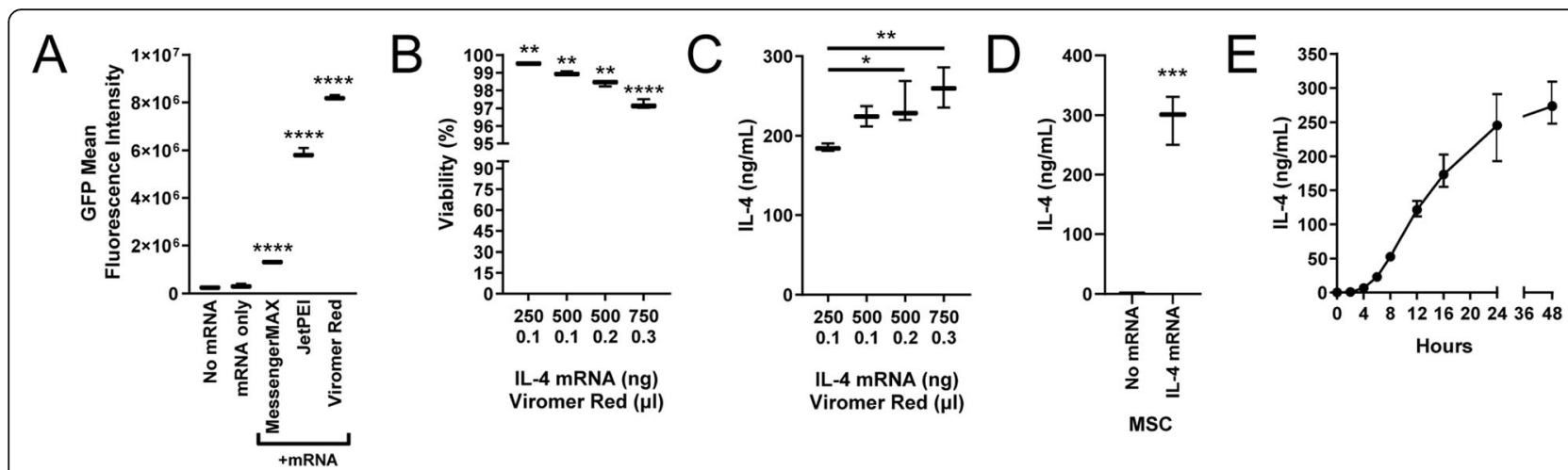

Fig. 1 Transfection of MSCs and protein expression. a GFP fluorescence intensity after MSCs are transfected with naked GFP mRNA or complexed with one of three vectors. $\mathbf{b}$ Viability of MSCs with increasing dosage of synthetic IL-4 mRNA complexed with Viromer Red. c Concentration of IL4 synthesized in media with IL-4 mRNA-transfected MSCs. d MSCs do not endogenously synthesize IL-4 and require transfection with IL-4 mRNA. e Concentration of IL-4 synthesized over $48 \mathrm{~h}$ from IL-4 mRNA-transfected MSCs. Mean \pm SD, one-way ANOVAs with Tukey's post hoc test, and Student's $\boldsymbol{t}$ test; ${ }^{*} p<0.05,{ }^{* *} p<0.01,{ }^{* *} p<0.001,{ }^{* * *} p<0.0001$

stimulator of the M2 cell-surface marker, CD206, on macrophages. We set up four groups: plain media, media conditioned by naïve MSCs (MSC CM), MSC CM supplemented with $100 \mathrm{ng}$ of recombinant IL-4 (rIL-4 CM), and media conditioned with IL-4 mRNA-transfected MSCs (synthesizing 80-100 ng expressed IL-4; eIL-4
CM) (Fig. 2a). We incubated the media with cultures of J774A.1 macrophages for $24 \mathrm{~h}$ after which time they were harvested, stained, and analyzed in a flow cytometer. Macrophage gating strategy (S. Fig. 2A) included fluorescence-minus-one (FMO) groups to distinguish positive staining (S. Fig. 2B). As hypothesized,

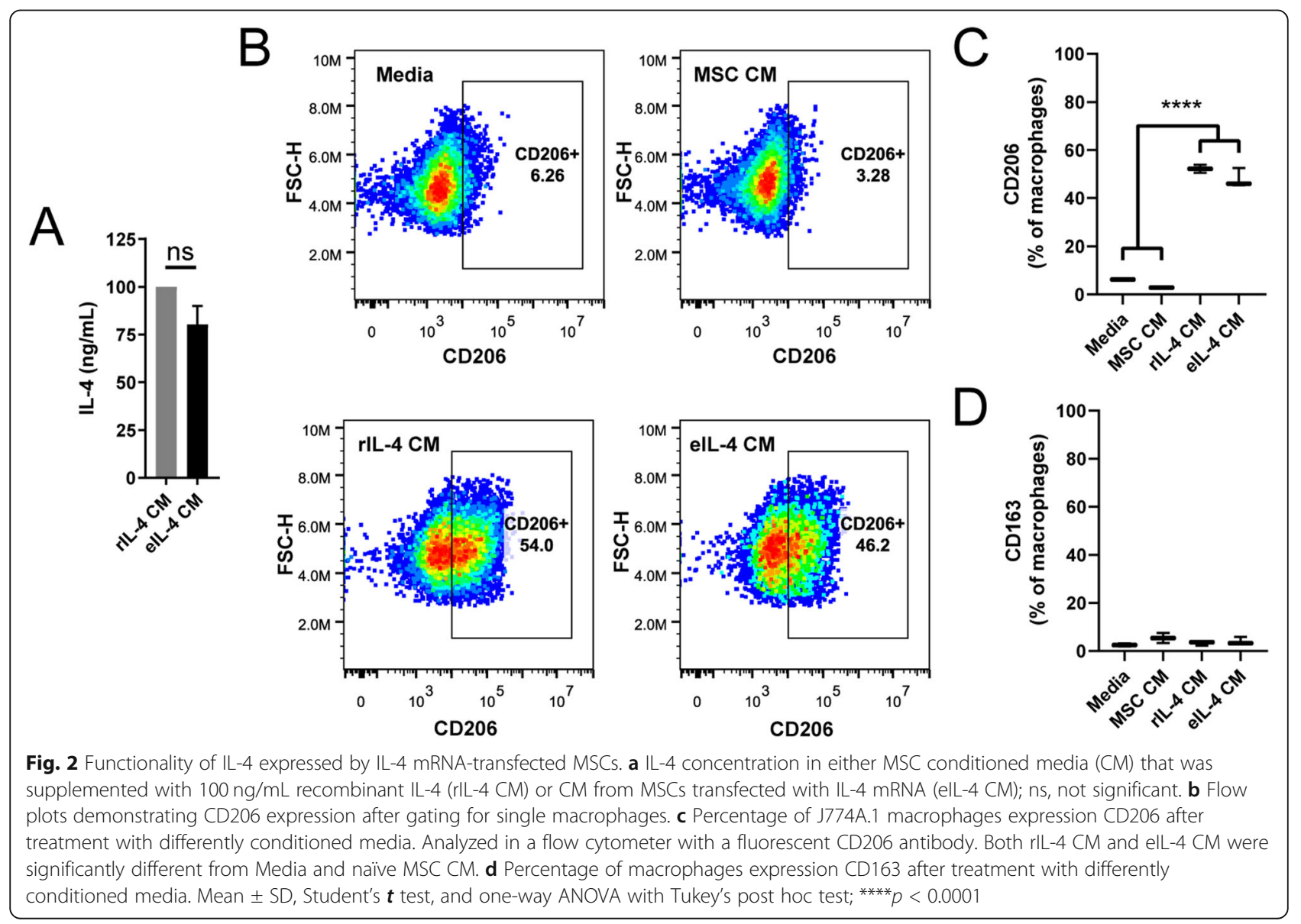


macrophages treated with either rIL-4 CM or eIL-4 CM demonstrated significantly greater expression of CD206 (Fig. 2b, c). As expected, CD163, an alternative M2-like marker (typically stimulated by IL-10), was not upregulated by IL-4 (Fig. 2d and S. Fig. 2C). Control experiments demonstrated that macrophage cell morphology and division rate was unaffected by media formulated for MSCs (S. Fig. 2D and E). To rule out the possibility of expressed IL-4 having an autocrine effect on MSCs, the MSCs were treated with $100 \mathrm{ng}$ of IL- 4 for $24 \mathrm{~h}$, washed, and CM was collected another $24 \mathrm{~h}$ later. When this $\mathrm{CM}$ was added to macrophages, there was no increase in CD206 expression (S. Fig. 2F), suggesting no autocrine effect of IL- 4 on MSCs. These studies demonstrated that IL-4 generated from synthetic IL-4 mRNAtransfected MSCs could induce an M2-like phenotype in macrophages.

\section{Transient MSC transfection is a viable strategy for in vivo experiments}

Due to the transience of IL-4 expression after mRNA transfection, it was critical to characterize expression considering the logistics of in vivo delivery. First, we set out to determine the ideal incubation time of IL-4 mRNA/Viromer Red complexes with MSCs to synthesize maximal IL4. MSCs were incubated with the complexes for varying durations $(0-24 \mathrm{~h})$. After this period, the cells were washed twice with PBS and left to express IL-4 in fresh media for $24 \mathrm{~h}$. Quantification of IL-4 via ELISA demonstrated that $8-12 \mathrm{~h}$ of transfection elicited the greatest IL4 production over the subsequent $24 \mathrm{~h}$ (Fig. 3a). Next, as multiple in vivo cell injections were expected to take 4-6 h, MSC survival on ice was assessed through trypan blue exclusion. Here, MSCs demonstrated $>95 \%$ viability over at least $6 \mathrm{~h}$ on ice (Fig. 3b). We also hypothesized that viability in media would be superior to that in PBS; however, we did not observe a significant difference. All in vivo injections were thus conducted in PBS to reduce variability. Curiously, media sampled from these transfected MSCs while on ice demonstrated minimal IL-4 expression (Fig. 3c). Then, regardless of how long the MSCs were on ice $(0-6 \mathrm{~h})$, the amount of IL-4 produced over the next $24 \mathrm{~h}$ in a $37^{\circ} \mathrm{C}$ incubator was equivalent (Fig. 3c). Finally, to scale for in vivo delivery, 300,000 cells per well were transfected for $10 \mathrm{~h}$ with either 1 or $2 \mu \mathrm{g}$ of IL-4 mRNA and a proportional amount of Viromer Red. Media was collected after $10 \mathrm{~h}$ prior to harvesting cells. After cells were harvested, 150,000 cells per well were re-plated. Media was collected again after $24 \mathrm{~h}$. The 300,000 cells transfected with $2 \mu \mathrm{g}$ induced at least $120 \mathrm{ng}$ of IL-4 prior to harvest. The harvested 150,000 cells then produced another $\sim 135 \mathrm{ng}$ of IL-4 over $24 \mathrm{~h}$ (Fig. 3d). This transfection protocol was chosen for all in vivo experiments; MSCs transfected thusly are henceforth denoted IL-4 MSCs.

\section{IL-4 MSCs induce an M2-like phenotype in vivo after brain injury}

To assess whether IL-4 MSCs can promote an M2-like phenotype of macrophages in vivo, IL-4 MSCs were injected in a TBI model of closed head injury (CHI) in mice. This model is similar to a model developed by Webster et al. but induces a motor deficit (described in methods and in Fig. 5) [80]. We injected either PBS, MSCs, MSCs expressing Luciferase (Luc MSCs), recombinant IL-4 (rIL-4), or IL-4 MSCs into the left hippocampus, 5 days post-injury (dpi). For groups containing MSCs, 150,000 cells were injected, a number based on prior studies [45]. Brains were harvested from euthanized mice 2 days after injection, 1 week post-injury
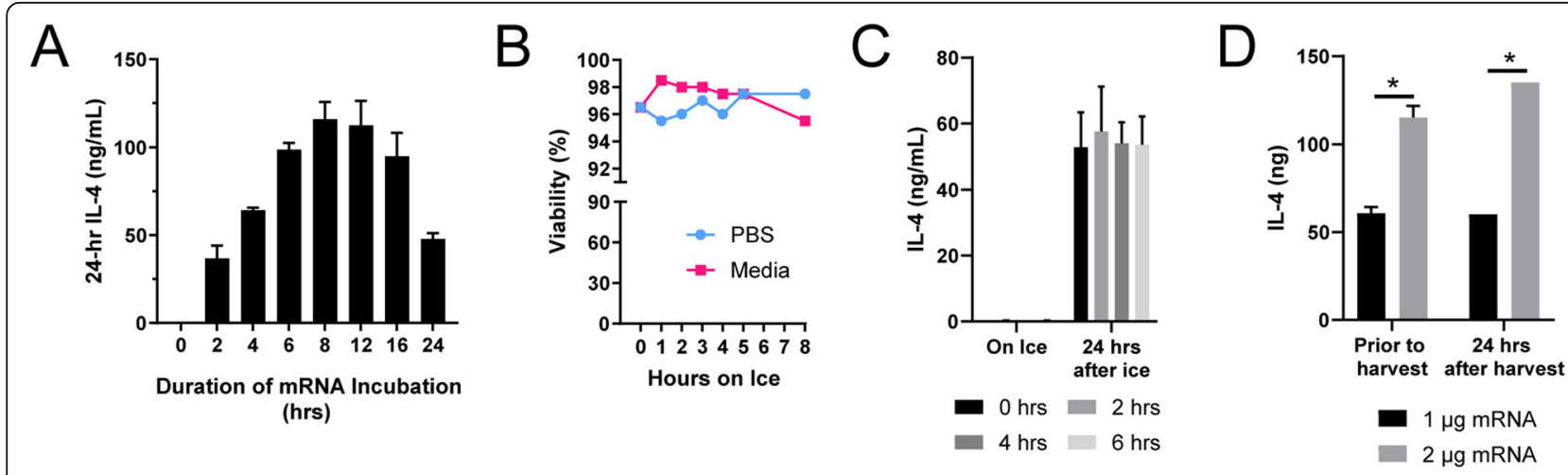

Fig. 3 MSC transfection characterization for in vivo delivery. a Concentration of IL-4 in media of MSCs transfected with synthetic IL-4 mRNA complexed with Viromer Red. The complexes were incubated with the MSCs for varying amounts of time (0-24 h). Then, the media was sampled $24 \mathrm{~h}$ after each time point for IL-4 quantification via ELISA. b Viability of MSCs while on ice. No significant differences observed. c Concentration of IL-4 synthesized while transfected and harvested MSCs were kept on ice (0-6 h) and then over $24 \mathrm{~h}$ after re-plating and kept at $37^{\circ} \mathrm{C}$. $\mathbf{d}$ Amount of IL-4 expressed by 300,000 MSCs transfected for $10 \mathrm{~h}$ ("prior to harvest") and then a sample of 150,000 MSCs $24 \mathrm{~h}$ after. Mean \pm SD and two-way ANOVA comparing between groups with Tukey's post hoc; ${ }^{*} p<0.05$ 


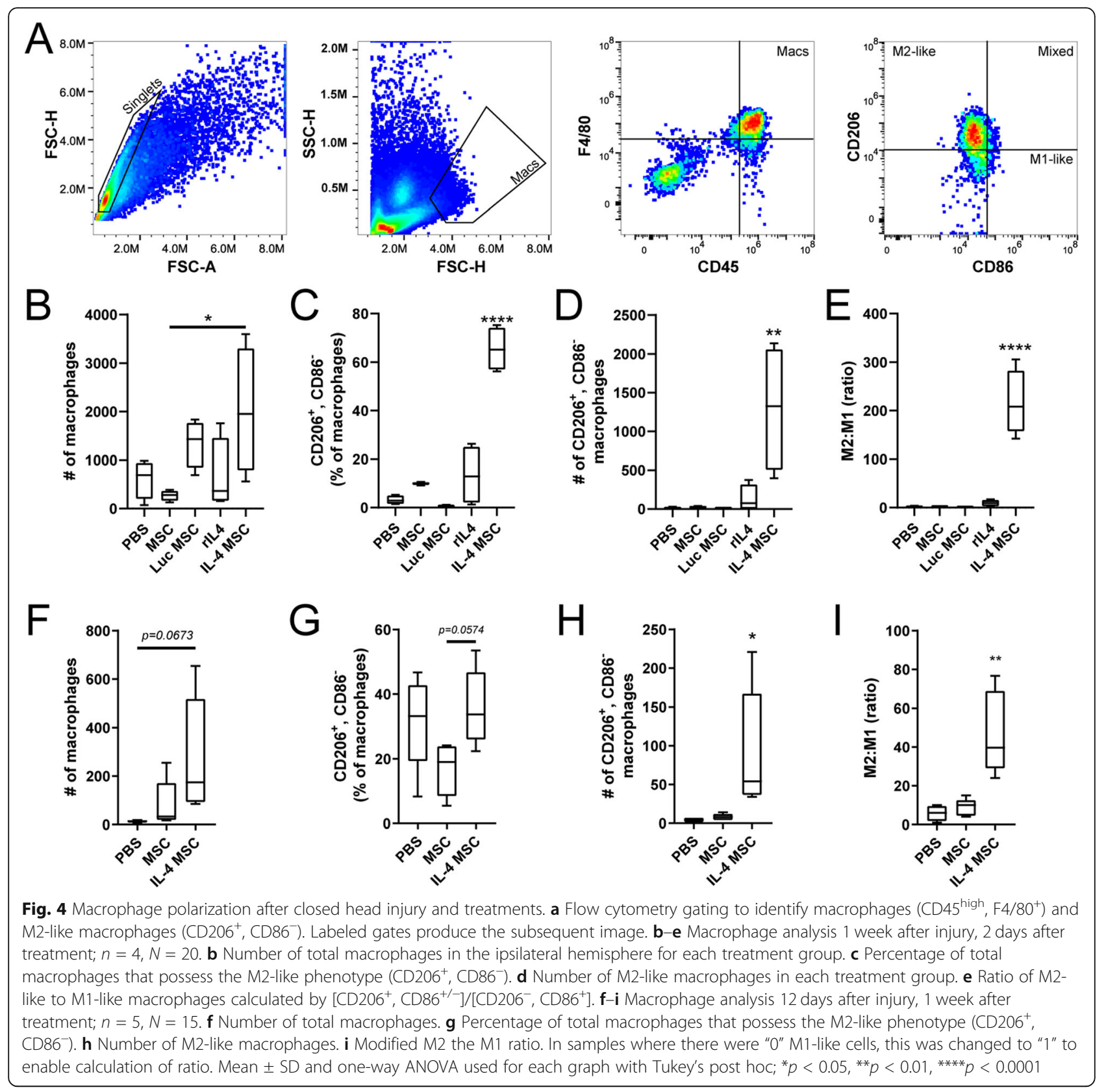

(wpi) and leukocytes isolated from the ipsilateral hemisphere were analyzed with flow cytometry (Fig. 4a). Leukocytes were gated via CD45 ${ }^{\text {high }}$ (S. Fig. 3A) [83], and FMO controls were used to determine the other antibody stains (S. Fig. 3B). First, we observed an increase in total macrophage $\left(\mathrm{CD} 45^{\text {high }}, \mathrm{F} 4 / 80^{+}\right)$number for most groups (Fig. 4b). Of these macrophages, IL-4 MSCs in-

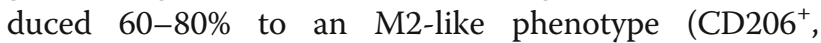
$\mathrm{CD}^{-} 6^{-}$(Fig. 4c). This was significantly higher than PBS $(<10 \%)$, MSCs alone $(20 \%)$, or Luc MSCs $(<10 \%)$. In comparison, an injection of $250 \mathrm{ng}$ of rIL-4 induced CD206 expression in 10-25\% of macrophages (Fig. 4c). IL-4 is known to induce proliferation of M2-like macrophages [94] and we observed that there were more $\mathrm{CD}_{206^{+}}$macrophages in the IL-4 MSC group compared to all other groups (Fig. 4d). We also calculated an M2 to M1 ratio: $\left[\mathrm{CD}_{206}{ }^{+}\right] /\left[\mathrm{CD} 206^{-}, \mathrm{CD}^{+} 6^{+}\right]$, a metric shown to be predictive of neural regeneration in a peripheral nerve injury model $[22,60]$ and correlated indirectly to improved outcomes in a TBI model [31]. IL-4 MSCs dramatically increased the M2 to M1 ratio compared to all other groups (Fig. 4e). While MSCs and rIL4 induced a higher percentage of M2-like macrophages than PBS (Fig. 4c), the M2 to M1 ratio showed no difference compared to PBS (Fig. 4e). An increase in the mRNA/Viromer Red transfection dose to MSCs greatly 
increased the number of M2-like macrophages (S. Fig. $3 \mathrm{C}$ and $\mathrm{E}$ ) while pushing the polarization only mildly higher to $80 \%$ (S. Fig. 3D). Interestingly, the M2 to M1 ratio was lower with this higher dose suggesting a low dose is more desirable (S. Fig. 3F).

Next, as CHI is diffuse, we required a delivery strategy that would affect the macrophages in both the ipsilateral (left) and contralateral (right) hemispheres. We hypothesized that delivering IL-4 MSCs into a lateral ventricle, instead of the hippocampus, would induce greater $\mathrm{CD}_{206^{+}}$macrophage expression in the contralateral hemisphere. Interestingly, the number of macrophages, mean percentage M2-like polarization, and number of M2-like macrophages in both ipsi- and contralateral hemispheres was lower with an intraventricular versus an intrahippocampal injection (S. Fig. 3G-I). Thus, all subsequent injections were made into the left hippocampus.

One week after treatment, the number of macrophages had fallen in all groups (Fig. 4f) compared to 2 days after injection (Fig. 4b). The M2-like phenotype (CD206 ${ }^{+}$, $\mathrm{CD}^{-} 6^{-}$) dropped to $\sim 37 \%$ of macrophages 1 week after IL-4 MSC injection (Fig. 4g). Intriguingly, $30 \%$ of macrophages in the PBS group retained an M2-like phenotype, while $\sim 20 \%$ of macrophages in the MSC only group did. However, the number of $\mathrm{CD}^{206^{+}}$macrophages was still significantly greater in the IL-4 MSC group compared to the others (Fig. 4h). A modified M2 to $\mathrm{M} 1$ ratio was calculated as some groups did not possess any $\mathrm{CD}^{206^{-}}, \mathrm{CD} 6^{+}$cells. This ratio was again significantly higher in the IL-4 MSC group compared to PBS and MSCs alone (Fig. 4i). These data demonstrate that IL-4 MSCs can increase the number of M2-like macrophages for at least up to 1 week after delivery and comprise $60-80 \%$ of all macrophages 2 days after injection. Although 1 week after intervention similar percentages of the M2-like phenotype exist regardless of treatment, a desired M2 the M1 ratio persists with IL-4 MSCs.

\section{Modified CHI model induces behavioral deficits that M2- like macrophage enrichment does not improve}

To assess whether our intervention has any effects on mouse behavior, we modified a published $\mathrm{CHI}$ model [80] to elicit motor and memory deficits. Severity of CHI was confirmed via time for the mouse to right itself from a supine position, as previous reports indicate a righting time (RT) $>10 \mathrm{~min}$ to indicate a severe injury [81]. Motor coordination/function was evaluated via persistence on an accelerating rotarod for at least 1 week after injury. Spatial learning and memory were using the Morris water maze (MWM), 4 weeks after injury. Our modified CHI model (with no injections) (Fig. 5a) was compared to mice receiving a sham surgical procedure: all experimental steps aside for the actual injury and injection. Injured mice demonstrated poorer rotarod performance in the first 2 weeks (Fig. 5b) and poor MWM outcomes 1 month out, compared to the sham group (Fig. 5c).

Next, we assessed whether the dramatic polarization and robust increase of $\mathrm{CD}_{206^{+}}$macrophages via IL-4 MSC treatment improved functional outcomes after CHI. Injured mice received either PBS or IL-4 MSCs 5 dpi into the left hippocampus. Rotarod assessment demonstrated no significant differences in performance between the two groups (Fig. 5d). A repeat of this experiment demonstrated similar results. MWM assessment demonstrated significant differences between the injured groups and sham, but no difference between IL4 MSCs and PBS (Fig. 5e). At baseline, injured mice that received an intrahippocampal injection took longer to find the hidden platform than sham and those who only received an injury (Fig. 5c, e). Finally, to test the hypothesis that an earlier change in macrophage phenotype could improve outcomes, we performed intrahippocampal injections 2 dpi. However, this also did not rescue acute changes in motor function (Fig. 5f). MWM was not repeated as Fig. 5e suggests the intrahippocampal injection itself affects performance. To confirm that RT was a reasonable measure for injury severity, a correlation analysis between RT and day 1 rotarod (D1RR) performance (as a proxy for severity) was performed. RT and D1RR demonstrated a moderate $(r=-0.53)$ and significant $(p<0.0001)$ correlation. In some trials, a more laborious severity assessment, Neurologic Severity Score (NSS) [82], was calculated; this had a stronger correlation $(r=-0.71)$ with D1RR. These studies suggest that our modified CHI model induces behavioral deficits and RT and NSS can confirm injury severity. Interestingly, enrichment of M2-like macrophages via IL-4 MSC treatment, does not improve functional outcomes.

\section{IL-4 MSCs increase IL-4 in both cerebral hemispheres and anti-inflammatory genes}

Given the conflicting M2-like polarization and behavioral data, we next explored how IL-4 MSCs affected inflammatory and anti-inflammatory cytokines and a select panel of genes. Injured mice received PBS, MSCs only, or IL-4 MSCs, and were compared to sham mice (no injury or injection). Multi-plex ELISA demonstrated that cerebral IL-4 levels were higher in the IL-4 MSC group 2 days after the intervention (1 wpi) compared to all other groups (Fig. 6a). This held true in both ipsiand contralateral hemispheres across the groups. Within the IL-4 MSC group, IL-4 levels were also significantly higher in the ipsilateral hemisphere $(0.45 \pm 0.34 \mathrm{pg} / \mathrm{mg})$ compared to the contralateral $(0.03 \pm 0.01 \mathrm{pg} / \mathrm{mg})$ (Fig. 6a). IL-6, a cytokine that is also secreted by M2-like 


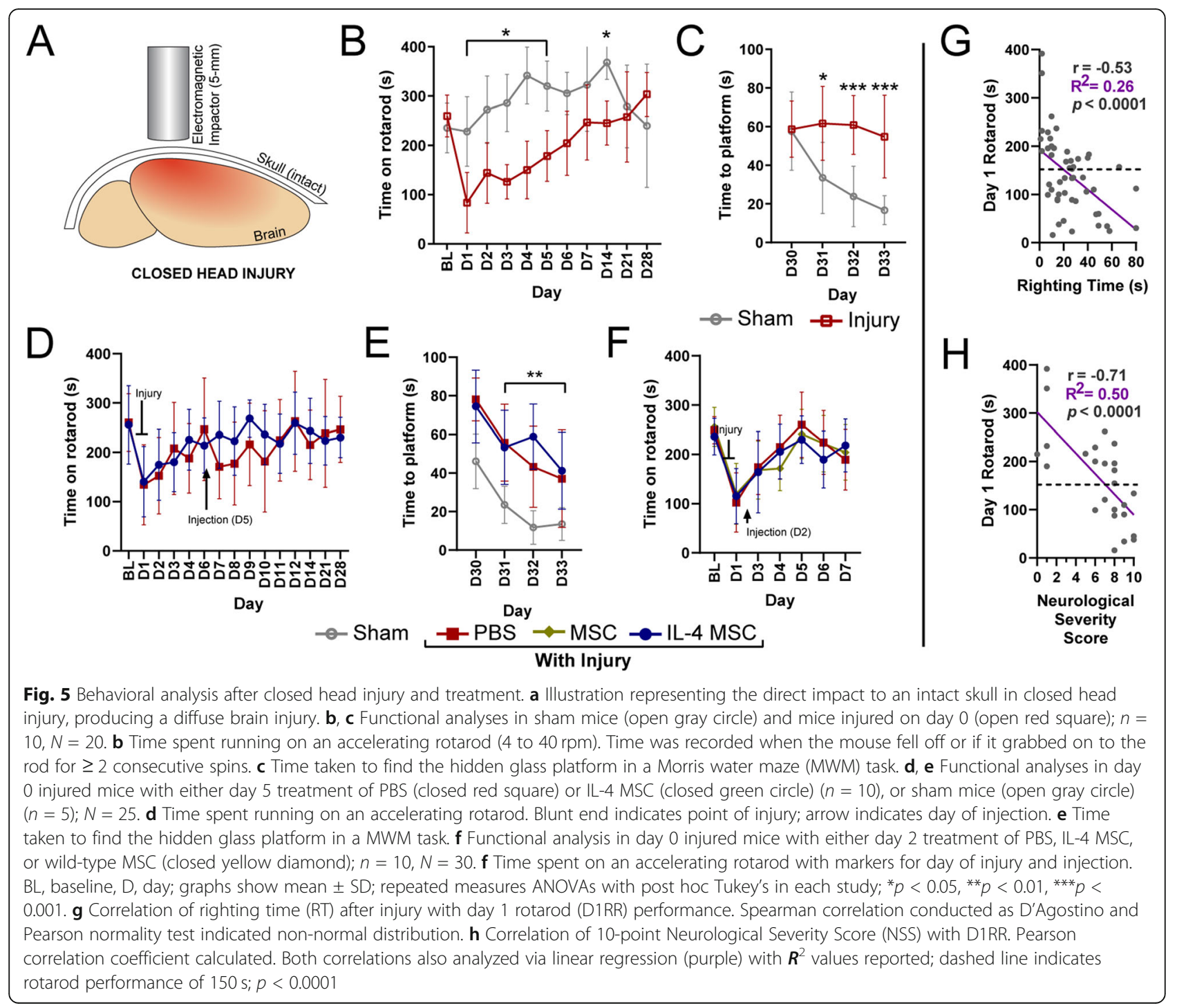

macrophages and known to have both beneficial and detrimental roles in TBI [95-98], was also significantly higher in the ipsilateral hemispheres of IL-4 MSCs mice compared to sham and PBS (Fig. 6b). There was no difference in IL-6 among the contralateral hemispheres (Fig. 6b). As anticipated, there were no significant differences in the other six inflammatory (S. Fig. 4A) and anti-inflammatory (S. Fig. 4B) cytokines studied among the four groups as cytokines tend to normalize to sham levels within a week after injury. A set of genes were investigated 1 and 3 wpi ( 2 and 16 days after treatment) through TaqMan Gene Expression Assays. $\Delta \Delta C \mathrm{~T}$ was calculated per group for each gene against all sham group mice. A selection of statistically significant genes is presented as a heatmap (Fig. 6c). The remaining nonsignificant gene assays, and fold changes of significant gene assays can be found in the supplement (S. Fig. 4C, S. Table 1). One week after injury, genes of two inflammatory cytokines (il1 $\beta, \quad c c l 2)$ and one antiinflammatory cytokine (illrn) were significantly upregulated at least $>2$-fold in all groups (PBS, MSC, IL-4 MSC) compared to sham mice (Fig. 6c and S. Table 1). However, il1rn (encoding the IL-1 receptor antagonist) was upregulated 20-25x in PBS and MSC groups while it was upregulated $85 \times$ in the IL-4 MSC group (S. Table 1). M2-like genes $m r c 1$ (encoding CD206) and $c d 163$ were elevated only in the IL-4 MSC group. Numerous genes assayed were also downregulated in all groups at this 1 wpi time point ( 2 days after treatment) including growth factors and genes related to axonal regeneration. Three weeks after injury, illrn upregulation had persisted in MSC and IL-4 MSC groups. Genes encoding growth factors and markers of neurogenesis tended to increase in expression at the 3-week time point; however, most of these did not reach statistical significance (S. Fig. 4C). The IL-4 MSC group also had statistically 


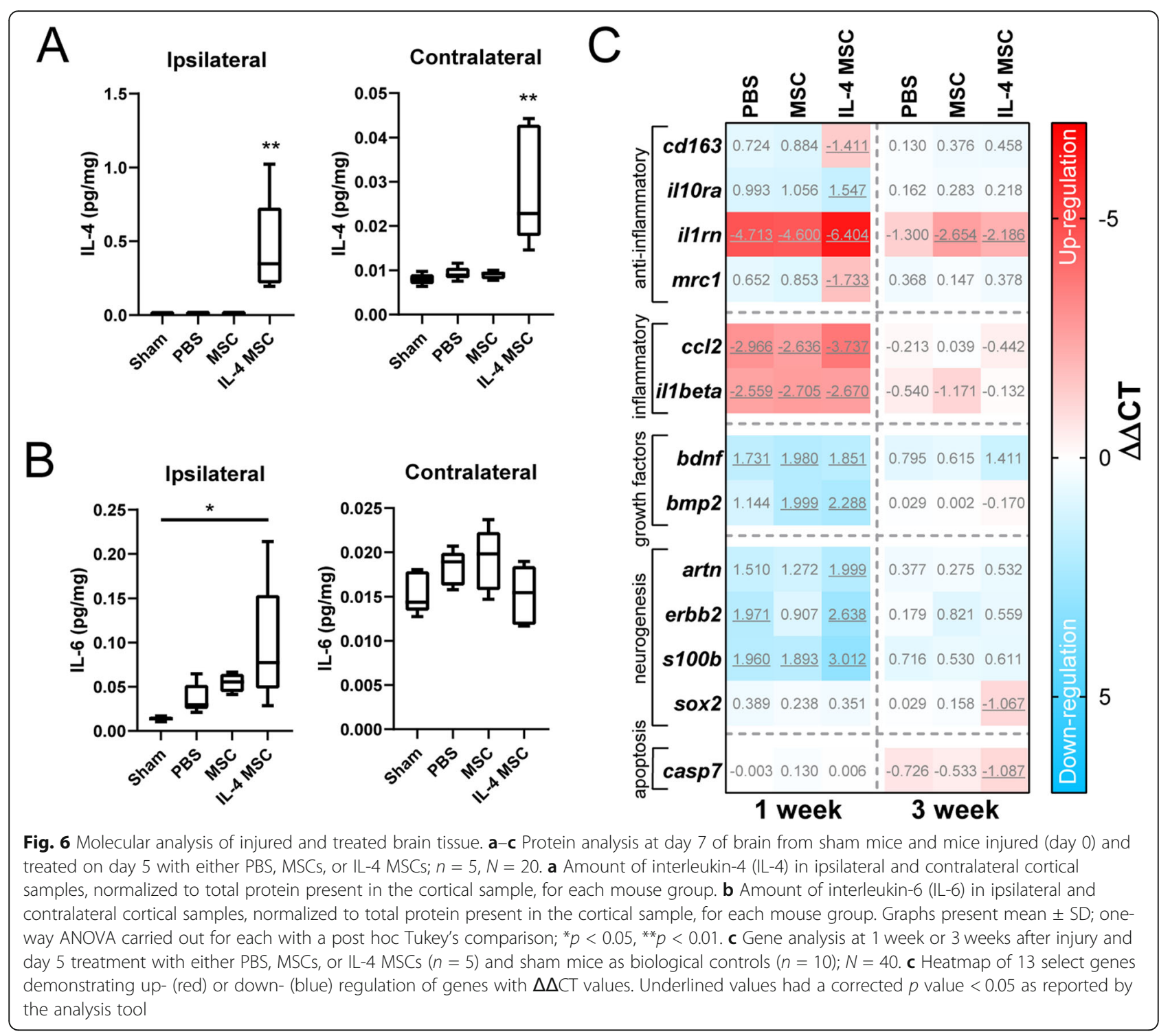

elevated sox2 (associated with neurogenesis or stem cells) and casp 7 (associated with apoptosis). These data suggest that while IL-4 MSCs acutely increase intracranial IL-4 levels and alters genes associated with inflammation, there is little to no long-term effect on genes associated with neurogenesis or growth factors.

IL-4 MSCs increase macrophages but these do not reduce astrogliosis or neuronal degeneration

We next scrutinized histological changes due to the absence of behavioral differences in the presence of increased M2-like macrophages. Markers for macrophages, astrogliosis, and neuronal degeneration were evaluated through immunohistochemistry (IHC) at 1 and 3 wpi ( 2 and 16 days after treatment) (Fig. 7 and S. Fig. 5). Mice were injured and treated with either PBS, MSCs, or IL-4 MSCs and compared to sham mice. Brain regions analyzed included ipsilateral and contralateral hippocampi, cortices, and the corpus callosum. Mean fluorescence was quantified through a semi-automated custom ImageJ script (Supplementary File 1). GFAP (a marker for reactive astrocytes) was elevated in all injured groups compared to sham (Fig. 7a) at both time points in both cortices (Fig. 7b, c and S. Fig. 5C). Expression of GFAP tended to be greater in the injected hemisphere (ipsilateral, left) at 1 wpi compared to the contralateral hemisphere and was also elevated in the ipsilateral hippocampus. However, there was no difference among the treatment groups. CD68 (a marker for macrophages) was absent in the sham group (Fig. 7d) but significantly elevated in all injury groups in both hemispheres (Fig. $7 \mathrm{e}-\mathrm{g}$ and S. Fig. 5D). CD68 expression was greater in the IL-4 MSC group compared to PBS in both the ipsilateral cortex and corpus callosum (Fig. 7f, g). CD68 ${ }^{+}$ 


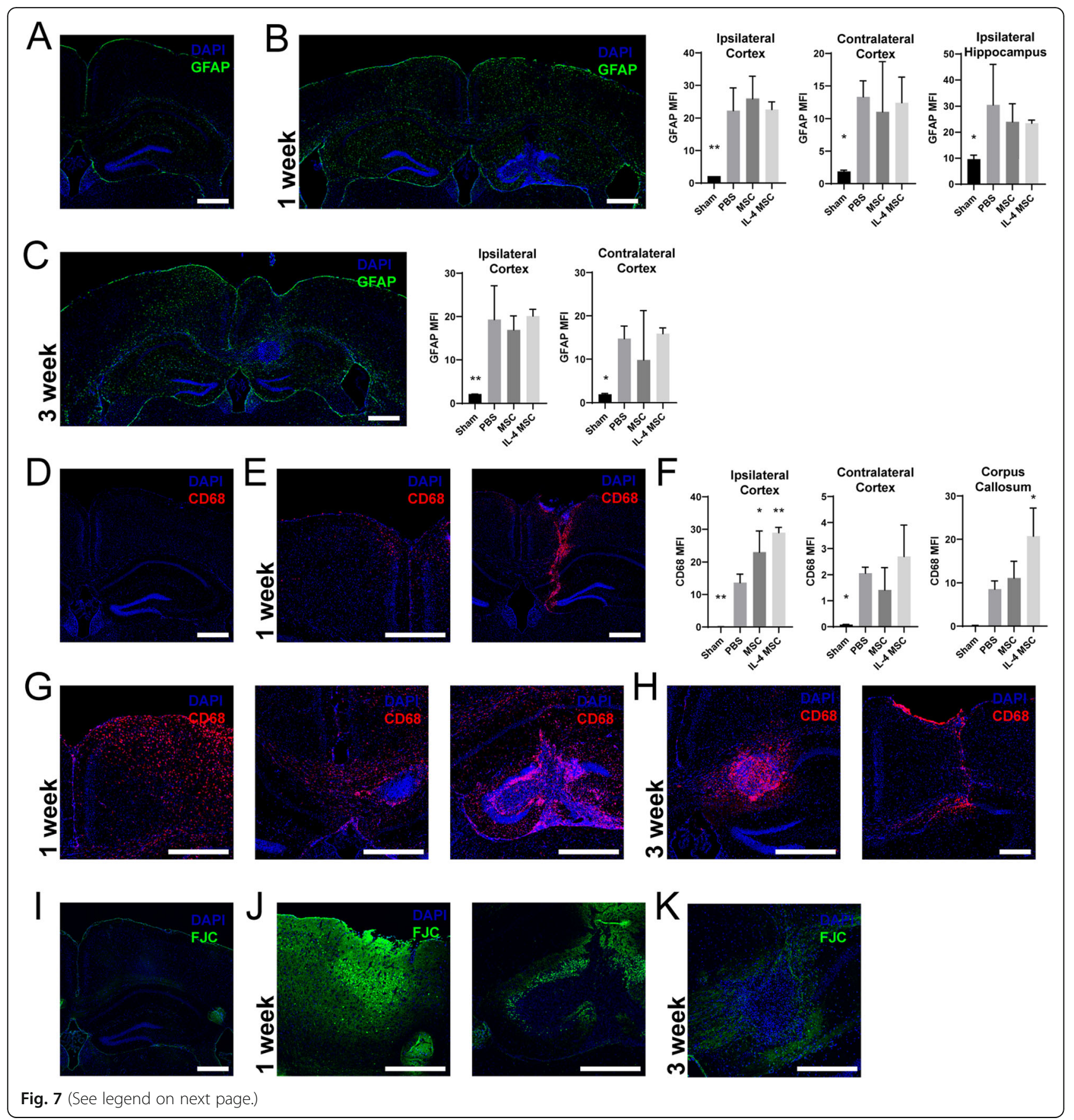


(See figure on previous page.)

Fig. 7 Immunohistochemical analysis of injured and treated brains at 1 and 3 weeks after injury. a Representative image of mouse brain section from sham group stained for GFAP (green) and DAPI (blue). b Representative image of mouse brain section from an injured group 1 week after injury. Image demonstrates diffuse reactive astrocytosis in both hemispheres. Graphs show mean fluorescence intensity (MFI) of GFAP in the ipsilateral and contralateral cortices, and the ipsilateral hippocampus for each group. c Representative image of a mouse brain section from an injured group 3 weeks after injury and stained for GFAP and DAPI. Graphs show GFAP MFI in the cortices for each group at this time point. $\mathbf{d}$ Representative image of brain section from the sham mouse group stained for DAPI (blue) and CD68 (red). e Representative sections from an injured mouse brain 1 week after injury who received PBS treatment on day 5. Left image shows macrophage infiltration in the contralateral cortex. Right image shows the extent of needle damaging the cortex and macrophage infiltration. $\mathbf{f}$ Graphs depict CD68 MFI across the treatment groups in both cortices and the corpus callosum. $\mathbf{g}$ Representative images from an injured mouse brain 1 week after injury who received IL-4 MSCs on day 5. Left image shows extensive macrophage infiltration in the left cortex. Middle image shows macrophage migration in the corpus callosum. Right image shows needle-induced damage and macrophage infiltration of the hippocampus. $\mathbf{h}$ Representative images from brains 3 weeks after injury. Left image is a section from a mouse treated with IL-4 MSCs. It shows a cluster of cells surrounded by macrophages. Right image is a section from a mouse treated with PBS. i Representative image of brain section from sham mouse group stained with DAPI and Fluoro-Jade C. $\mathbf{j}$ Representative images 1 week after brain injury. Left images demonstrate neuronal degeneration in the cortex. Right image shows neuronal degeneration in the hippocampus. $\mathbf{k}$ Representative image 3 weeks after brain injury with less neuronal degeneration than after the first week. Graphs shows mean \pm SD; one-way ANOVAs with Dunnett's multiple comparison against the PBS group. ${ }^{*} p<0.05,{ }^{* *} p<0.01$

cells were also seen across the corpus callosum and in the contralateral hemisphere (Fig. $7 \mathrm{~g}$ ). We also observed dramatic structural alterations in the ipsilateral hippocampus caused by the injection needle (Fig. 7e, g). At 3 wpi, in the MSC and IL-4 MSC groups, a cluster of cells was often found surrounded by macrophages (Fig. 7h, left). MSCs were absent in the PBS group, but staining nonetheless indicated presence of macrophages and microglia (Fig. 7h, right). Fluoro-Jade $\mathrm{C}$ staining demonstrated no neuronal degeneration in sham mice (Fig. 7i) but was detectable in all injured groups at both time points (Fig. 7j, k and S. Fig. 5E). Greater staining was visible in the cortices and hippocampus at $1 \mathrm{wpi}$ (Fig. $7 \mathrm{j}$ and S. Fig. 5E) than at 3 wpi. These histological
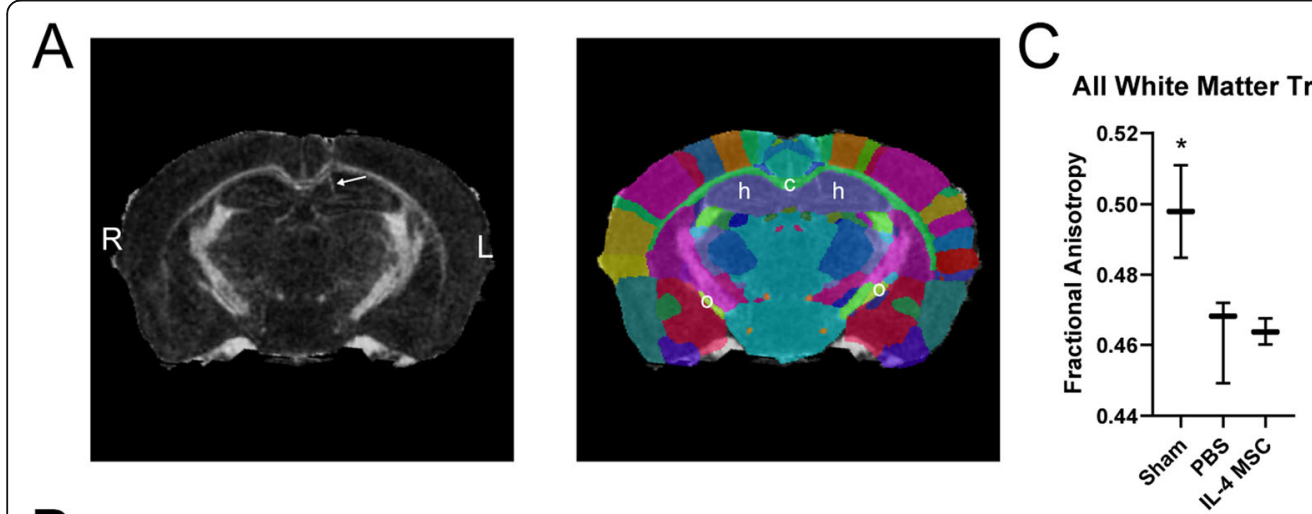

Brachium of the
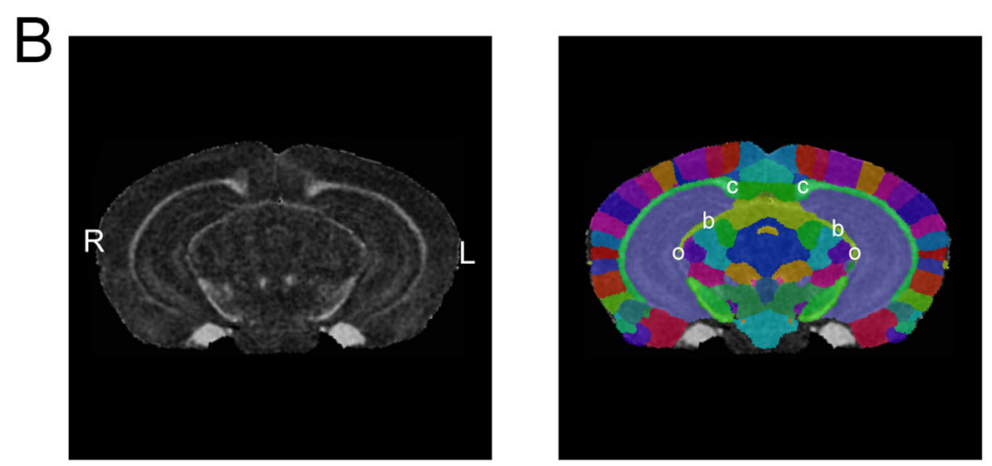

Corpus Callosum

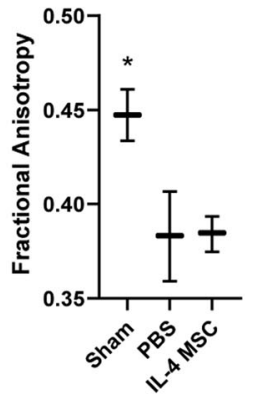

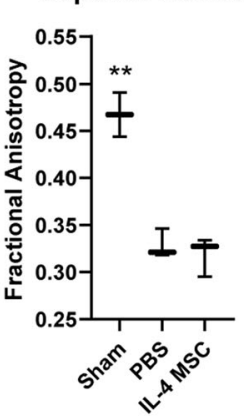

Optic Tracts

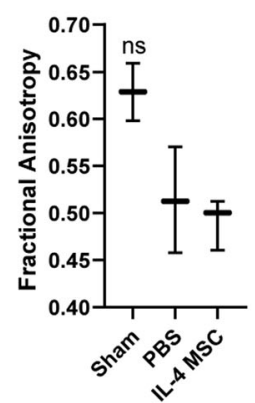

Fig. 8 Diffusion-weighted magnetic resonance imaging to assess white matter integrity 1 month after injury. $\mathbf{a}$, $\mathbf{b}$ Left images are representative coronal brain slices of fractional anisotropy. Right images are corresponding map of cortical regions. Arrow indicates injection artifact. b, brachium of the superior colliculus; c, corpus callosum; h, hippocampus; o, optic tract. c Fractional anistropy values of each region per treatment group. Sham $n=2$, PBS $n=3$, IL-4 MSCS $n=3, \boldsymbol{N}=8$; graphs present mean \pm SD; one-way ANOVAs with post hoc Dunnett's multiple comparison against the PBS group; ns, not significant, ${ }^{*} p<0.05,{ }^{* *} p<0.01$ 
assessments demonstrate that IL-4 MSCs recruit numerous macrophages, but those macrophages fail to reduce the diffuse astrocytosis or neuronal degeneration our CHI model induces.

\section{IL-4 MSC recruited macrophages do not improve white matter integrity after injury}

In brain repair, M2-like macrophages are associated with improved white matter integrity (WMI) [24, 25]. To assess whether the M2-like phenotype induced by IL-4 MSCs improved WMI after TBI, a subset of injured mice treated with PBS or IL-4 MSCs and a subset of sham mice were scanned 5 wpi with diffusion-weighted MRI (Fig. 8a, b). The images were mapped to a dataset to automatically orient and identify white matter regions of interest (Fig. 8a, b). Values of fractional anisotropy (FA) and diffusivity (axial, radial, and mean) were obtained for each region. White matter tracts are anisotropic and when subjected to trauma, this anisotropy is reduced $[99,100]$. Thus, we hypothesized that our injury would also disrupt white matter tracts and thus reduce FA while increasing diffusivity. Compared to sham, FA values were lower in the PBS and IL-4 MSC groups in all white matter tracts; however, no difference was observed between the two treatments (Fig. 8c). FA was particularly reduced in the brachium of the superior colliculus, corpus callosum, and optic tracts (Fig. 8c) of both injured and treated groups. While there were trends in diffusivity measures, differences between groups were not statistically significant. This MRI study demonstrated that while injury reduced WMI, M2-like macrophage enrichment via IL-4 MSCs did not improve WMI 1 month after delivery.

\section{Transcriptomics elucidates persistent inflammation and poor regeneration}

To develop a mechanistic understanding of the link between elevated M2-like macrophage polarization and most measures of wound healing, we investigated the transcriptome at the treatment area using RNA-seq at 1 and 3 wpi (Fig. 9). To contextualize the data, overrepresented pathways were consolidated (S. Table 2 and 4), and significantly enriched pathways were separated into pathway groups (S. Table 3). Overall, IL-4 MSCs induced more significantly upregulated genes at 1 wpi than any other group (Fig. 9a). However, this upregulation was absent at 3 wpi (Fig. 9a).

As expected, numerous immune response and inflammation pathways were upregulated acutely by all groups compared to sham. However, although leukocyte numbers and inflammatory cytokines were reduced in prior data by 3 wpi (Fig. 4 and 6), inflammation-associated pathways and genes were still upregulated here (Fig. 9bd). Specifically, cytokine, inflammatory interleukin, $\mathrm{T}$ cell, and macrophage pathway groups were chronically upregulated by MSCs compared to PBS, but this effect was absent in the IL-4 MSC group (Fig. 9c). Pathways related to IL-4 were enriched by IL-4 MSCs compared to PBS and MSCs at 1 wpi (Fig. 9c). However, these pathways were not enriched at 3 wpi, suggesting that the IL-4 response does not persist. Ultimately, while fewer inflammatory pathways were over-represented by IL-4 MSCs compared to MSCs, general inflammation pathways were enriched and genes upregulated by all groups compared to sham at 3 wpi.

Alongside persistent inflammation, pathways of axonogenesis were over-represented at $1 \mathrm{wpi}$ (Fig. 9b) in Sham, MSC, and PBS groups, and enriched (Fig. 9c) in MSC and PBS groups compared to IL-4 MSCs at 1 wpi. One of these, "GO_NEGATIVE_REGULATION_OF_AXONOGENESIS" (S. Table 3), also suggests downregulation of axonogenesis in both groups relative to IL-4 MSCs. At $3 \mathrm{wpi}$, the pathway, "GO_NEUROGENESIS," was enriched in MSCs compared to IL-4 MSCs (Fig. 9c). Regarding myelination, few related pathways were enriched among injury and treatment groups, but at 3 wpi oligodendrocyte pathways were enriched compared to sham (Fig. 9c). Glial pathways were found to be enriched at 1 wpi for IL-4 MSCs, and in contrast, 3 wpi for MSCs (Fig. 9c). Thus neural regeneration, myelination, and gliosis did not improve from treatment.

At 1 wpi, IL-4 MSCs exhibited enrichment of regeneration, healing, stem cell, and angiogenesis pathway groups relative to MSCs and PBS (Fig. 9c). However, at $3 \mathrm{wpi}$, MSCs tended to have higher normalized enrichment scores for these groups compared to IL-4 MSCs (Fig. 9c). IL-4 MSCs also exhibited enrichment of endothelial and platelet derived growth factor-related pathways at $1 \mathrm{wpi}$, while MSCs showed enrichment of fibroblast and insulin-like growth factor-related pathways at 3 wpi (Fig. 9c).

All injury groups over-represented a "response to other organism" pathway, suggesting similar responses between injury and infection (Fig. 9b). The PBS group at 3 wpi had over-representation for neurofibrillary tangles and tau protein-related pathways compared to Sham, suggesting some overlap in TBI response to that seen in Alzheimer's disease (S. Table 2). At 3 wpi, IL-4 MSCs and MSCs had over-represented pathways suggesting autoimmune activation, including graft-versus-host disease and allograft rejection (Fig. 9b and S. Table 2).

To begin to understand effects on microglia, we performed over-representation analysis of our gene data against a database that clustered microglia genes based on different stimulation conditions (S. Table 4). As expected, a cluster representing IL-4 stimulation of microglia ("SA_Salmon") was over-represented in the IL-4 MSC group at 1 wpi compared to both PBS and MSC. 

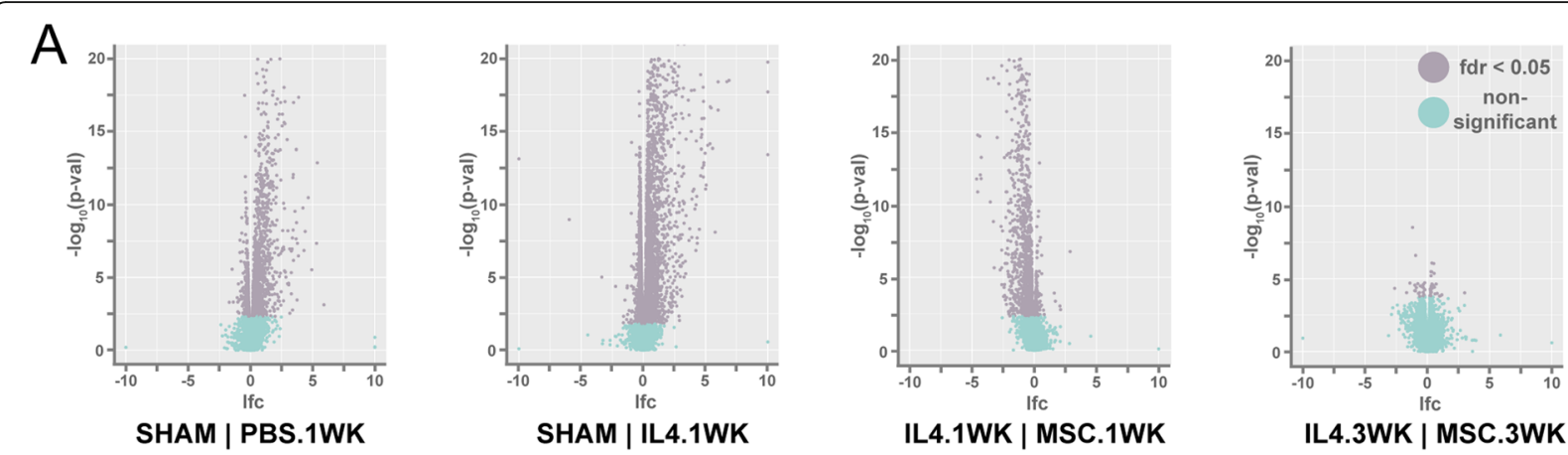

B

Select Significantly Overrepresented Pathways (g:Profiler)

\begin{tabular}{llll}
\hline Ontology & Pathway & Group & Comparison \\
\hline GO:0002376 & immune system process & IL-4 MSC (1 \& 3 WK) & SHAM, PBS 1WK, MSC 1WK \\
\hline GO:0051707 & response to other organism & IL-4 MSC (1 \& 3 WK) & SHAM, PBS 1WK, MSC 1WK \\
\hline KEGG:05330 & allograft rejection & IL-4 MSC (3 WK), MSC (3 WK) & PBS 3WK \\
\hline GO:0001525 & angiogenesis & IL-4 MSC (1 WK) & SHAM, PBS 1WK, MSC 1WK \\
\hline GO:0007409 & axonogenesis & SHAM, PBS (1WK), MSC (1 WK), IL-4 MSC (3WK) & IL-4 MSC (1 WK)
\end{tabular}

C

Select Significantly Enriched Pathways (GSEA)

\begin{tabular}{|c|c|c|c|c|c|}
\hline Category & Example Pathway & Week(s) & Enriched Group & Comparison & NES \\
\hline Inflammation & GO_NEUROINFLAMMATORY_RESPONSE & $1 \& 3 \mathrm{WK}$ & PBS, MSC, IL-4 MSC & SHAM & $1.46-2.10$ \\
\hline Inflammation & GO CYTOKINE SECRETION INVOLVED IN IMMUNE RESPONSE & $3 \mathrm{WK}$ & PBS, MSC & IL-4 MSC & $1.76-1.95$ \\
\hline Inflammation & GO_RESPONSE TO_INTERLEUKIN_ 2 & $3 \mathrm{WK}$ & MSC & PBS, IL-4 MSC & $1.55-2.10$ \\
\hline Inflammation & GO_INTERLEUKIN_4_PRODUCTION & $1 \mathrm{WK}$ & IL-4 MSC & PBS, MSC, IL-4 MSC (3WK) & $1.47-1.56$ \\
\hline Immune & GO_T_CELL_ACTIVATION & $3 \mathrm{WK}$ & MSC, IL-4 MSC & PBS & $1.89-2.47$ \\
\hline Myeloid & GO_MACROPHAGE_ACTIVATION & $3 \mathrm{WK}$ & MSC & PBS, IL-4 MSC & $1.52-1.96$ \\
\hline Glial & GO_GLIAL_CELL_ACTIVATION & $1 \mathrm{WK}$ & IL-4 MSC & PBS, MSC & $1.58-1.63$ \\
\hline Glial & GO_GLIAL_CELL_ACTIVATION & $3 \mathrm{WK}$ & MSC & PBS & 1.55 \\
\hline Myelination & GO_OLIGODENDROCYTE_DEVELOPMENT & $3 \mathrm{WK}$ & PBS, MSC, IL-4 MSC & SHAM & $1.52-1.70$ \\
\hline Myelination & GO STRUCTURAL_CONSTITUENT_OF_MYELIN_SHEATH & $1 \mathrm{WK}$ & MSC & PBS, IL-4 MSC & $1.56-1.71$ \\
\hline Neuronal & GO_REGULATION OF_AXONOGENESIS & $1 \mathrm{WK}$ & PBS, MSC & IL-4 MSC & $1.49-1.77$ \\
\hline Neuronal & GO_NEUROGENESIS & $3 \mathrm{WK}$ & MSC & IL-4 MSC & 1.55 \\
\hline Regeneration & GO_TISSUE_REGENERATION & $1 \mathrm{WK}$ & IL-4 MSC & PBS, MSC & $1.54-1.67$ \\
\hline Regeneration & GO_WOUND_HEALING & $1 \mathrm{WK}$ & IL-4 MSC & PBS, MSC & $1.49-1.53$ \\
\hline Regeneration & GO_WOUND_HEALING & $3 \mathrm{WK}$ & MSC & PBS, IL-4 MSC & $1.55-1.65$ \\
\hline Regeneration & $\begin{array}{l}\text { GO_VASCULAR_ENDOTHELIAL } \\
\text { GROWTH_FACTOR_PRODUCTION }\end{array}$ & $1 \mathrm{WK}$ & IL-4 MSC & PBS, MSC & $1.53-1.61$ \\
\hline Regeneration & GO_RESPONSE_TO_FIBROBLAST_GROWTH_FACTOR & $3 \mathrm{WK}$ & MSC & IL-4 MSC & 1.65 \\
\hline Stem Cell & $\begin{array}{l}\text { GO_POSITIVE_REGULATION_OF_NEURAL_PRECURSOR_ } \\
\text { CELL_PROLIFERATION }\end{array}$ & $1 \& 3 \mathrm{WK}$ & PBS, MSC, IL-4 MSC & SHAM & $1.40-1.68$ \\
\hline Stem Cell & GO_HEMATOPOIETIC_STEM_CELL_DIFFERENTIATION & $1 \mathrm{WK}$ & IL-4 MSC & PBS, MSC & $1.43-1.54$ \\
\hline Stem Cell & GO_HEMATOPOIETIC_STEM_CELL_PROLIFERATION & $3 \mathrm{WK}$ & MSC & IL-4 MSC & 1.61 \\
\hline
\end{tabular}

D anti-inflammatory neurogenesis

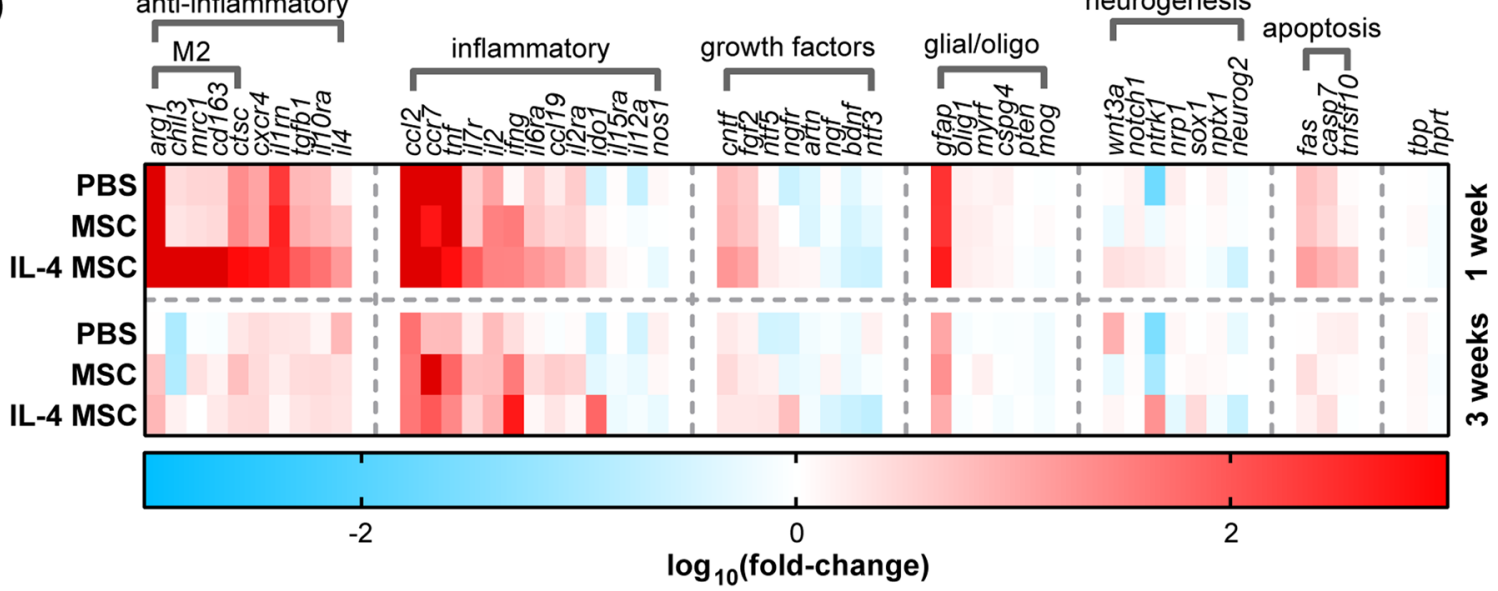

Fig. 9 Transcriptomic analysis of mouse brains local to site of intervention at 1 and 3 weeks. a Volcano plots demonstrating that IL-4 MSCS induced the most alterations in gene expression. Each graph presents genes of the latter condition vs. the former condition. $\mathbf{b}$ Select significantly over-represented pathways identified through g:Profiler and reported in S. Table 1. c Select significantly enriched pathways identified through Gene Set Enrichment Analysis and Molecular Signatures Database v7.0, Collection C5, reported in S. Table 2. d A heatmap presenting a selection of genes up- or downregulated compared to the Sham group 
However, a few other clusters representing interferon (IFN) and lipopolysaccharide (LPS) stimulation were also over-represented at 1 wpi. At 3 wpi, both MSCs and IL-4 MSCs had over-represented clusters representing IFN and LPS stimulation compared to sham (BR_Blue, BR_ Turquoise, PI_Turquoise). However, among the injury groups, there were little differences. IL-4 MSCs had one cluster over-represented compared to PBS (BR_Turquoise) that suggested a response analogous to persistent IFN $\gamma$ and LPS stimulation.

Overall, our analyses suggest that (1) acute benefits of IL-4 MSCs do not persist, (2) inflammation persists to at least 3 weeks even with treatment, and (3) growth factor expression, neural regeneration, and myelination does not increase with treatment.

\section{Discussion}

One important reason a patient suffers disability after TBI is because the immunological response becomes maladaptive. To shift the immune response toward a regenerative phenotype, clinical trials are investigating therapeutic stem cell delivery. However, these stem cells do not secrete some potent regulators of inflammation. One absentee, IL-4, is a strong inducer of the antiinflammatory, M2-like phenotype in macrophages and microglia. According to the literature, M2-like macrophages are associated with improved biological and functional outcomes in pre-clinical TBI models. Thus, in this study, we augmented MSCs to transiently express IL-4 and consequently observed a robust increase in M2-like macrophages and some anti-inflammatory genes after TBI. Curiously, this biological change did not improve functional or histological outcomes, though our transcriptomic analyses provide some potential answers as to why not.

We began this work by transiently expressing IL-4 from MSCs and studying in vivo IL-4 levels and M2-like macrophage polarization. IL-4 has been welldocumented as an inducer of this phenotype in macrophages and microglia in vivo in CNS and PNS injury models. In fact, transfection of macrophages with IL-4 mRNA also promotes an M2 phenotype in vitro and at least M2-like in vivo [79]. Our results corroborated this as we saw a dramatic rise in the polarization and quantity of M2-like macrophages at both 2 and 7 days after our intervention (about 1 and 2 wpi). Simultaneously, we witnessed upregulation of numerous anti-inflammatory and M2-like genes. However, while this polarization may be desirable, IL-4 induces macrophage proliferation as well. Thus, it might be important to understand how much IL-4 is required in vivo and how many M2-like macrophages are optimal for neuroprotection. Our data begin to answer some of these questions. We saw that a bolus of recombinant IL-4 is less effective at polarizing macrophages than sustained expression over $24-48 \mathrm{~h}$ from IL-4 MSCs (Fig. 4). Separately, we observed that a small proportion of IL-4 from IL-4 MSCs reaches the contralateral hemisphere (Fig. 6a). Surprisingly, this induced comparable M2-like polarization as in the ipsilateral hemisphere (S. Fig. $3 \mathrm{H}$ ). Critically, however, the total number of macrophages and specifically M2-like macrophages was significantly lower in the contralateral hemisphere (S. Fig. 3G and I). Finally, 1 week after intervention, IL-4 MSCs still had a greater number of M2like macrophages compared to other groups, though the polarization percentage had dropped. Most of the macrophages did not express CD206 or CD86, suggesting they may possess a phenotype we did not explore. Our transcriptomic data also demonstrated a signature for persistent immune and inflammatory response in all injured groups compared to sham. Furthermore, IL-4 response-related pathways did not persist with IL-4 MSC treatment (Fig. 9c). Thus, hypothetically, an ideal scenario would recruit much fewer macrophages, with the majority biased to be M2-like. These data suggest that a sustained, yet smaller dose of endogenous IL-4 could foster that.

Second, we tested our IL-4 MSCs in a TBI model of $\mathrm{CHI}$ but did not see an acute improvement in motor coordination or memory. Numerous CHI models exist, and these have been extensively reviewed [101]. We believe that since $\mathrm{CHI}$ induces a diffuse injury, it is more clinically relevant than focal TBI models. Studying behavioral outcomes, however, can be tricky. The central understanding is, if function is improved, the therapy had long-reaching effects; it not only influenced biology directly, but ultimately also promoted neuroprotection, regeneration, or plasticity. However, TBI models have high intrinsic biological and technical variability (Fig. 5g, h). Thus, for all TBI studies assessing behavior, it becomes critical to (1) demonstrate that injury induced equivalent dysfunction in all groups and (2) use statistics that correct for repeated measures. We modified a published $\mathrm{CHI}$ model by increasing parameter severity [80] to induce a significant behavioral deficit (Fig. 5b, c). A correlation analysis between rotarod performance, D1RR, and two post-injury tests (RT and NSS) indicated moderate to strong correlation, suggesting future studies could further fine-tune the severity of injury (Fig. $5 \mathrm{~g}$, h). However, intervening with IL-4 MSCs did not improve rotarod performance or memory (Fig. 5d-f). Unfortunately, this corroborates many other attempts with genetically modified MSCs [52, 66, 68, 70]. Studies that saw an improvement typically saw a change in only one out of multiple assessments, or only looked at a single time point $[66,67,69]$. In one study delivering recombinant IL-4 after stroke in IL-4 knockout mice, repeated measures were not accounted for and wild-type mice were 
not tested [55]. A variety of other approaches to TBI that promote an M2-like response have also observed improvement in behavioral outcomes [26-41]. However, in many of these studies, behavior was not (or could not be) assessed prior to treatment; thus, procedural and measurement biases cannot be ruled out. In this study, we also only looked at 1 and $3 \mathrm{wpi}$; it is conceivable that the M2-like macrophages might have limited the spread of damage. Benefits of this might only be seen over months as neural rewiring slowly takes place. Interestingly, our transcriptomic analysis of IL-4 MSCs demonstrated that pathways relating to tissue regeneration and healing were enriched at 1 wpi (Fig. 9c). However, pathways relevant to neural regeneration (e.g., axonogenesis, neurogenesis) were over-represented or enriched in the other groups instead (Fig. 9b, c). Thus, our data suggest that either M2-like macrophage enrichment alone via IL-4 MSC intervention could not alter function, that our behavioral assessments may not have been adequately sensitive to reveal the effects of IL-4 MSCs on TBI, or that the severity of the induced injury was too great.

Third, while injury alone induced histological changes, we observed damage caused by intrahippocampal injections as well. Histology demonstrated significant increase in GFAP across all injured groups. GFAP is a marker for reactive astrocytosis, an endogenous response to limit deleterious effects of TBI. However, chronically, astrocytosis also prevents neural regeneration. Interestingly, transcriptomic analysis suggested that gliosis pathways were only enriched acutely in the IL-4 MSC group compared to PBS and MSC groups (Fig. 9c), a potentially desirable scenario. At $3 \mathrm{wpi}$, it was MSCs that had gliosisrelated pathways enriched compared to IL-4 MSCs (Fig. 9c). However, both the amount of gliosis observed histologically (Fig. 7c), and the transcriptomic enrichment of gliosis pathways compared to sham suggest that IL-4 MSCs do not reduce gliosis in a biologically relevant manner within 3 weeks. IL-4 MSCs did, however, increase the number of macrophages (indicated by CD68 staining and corroborated by flow cytometry). However, the needle used to inject the treatments, and possibly the bolus of cells, negatively impacted the architecture of the ipsilateral hippocampus (Fig. 7g). We chose intrahippocampal delivery over intraventricular due to the improved polarization (S. Fig. 3H). Nevertheless, this likely reduced memory performance in the MWM task (Fig. 5e). Interestingly, one TBI clinical trial is exploring stereotactic intracerebral delivery of modified MSCs (NCT02416492). However, an intraventricular delivery would have saved the hippocampus and likely left a smaller cortical injury as the cerebral spinal fluid and ventricles can better accommodate a transiently increased volume. In our studies, we did not assess intravascular delivery as $<0.1 \%$ of MSCs cross the BBB [102] even though it may be permeable soon after TBI [103]. However, this may still be a viable approach. In this case, instead of targeting IL-4 MSCs toward the brain, the modified MSC secretome could influence peripheral monocytes prior to invading the TBI environment. In fact, many of the TBI interventions that improved behavioral outcomes were through intravascular routes. Interestingly, IL-10 improved outcomes when administered intravenously but not when delivered intracranially [104]. This difference in effect has also been observed with cord blood cells for stroke [105]. Thus, our data and the literature suggest that systemic delivery may be the better avenue for TBI even though the BBB is generally considered an obstacle.

Finally, our studies also suggest that $\mathrm{CD} 206^{+}, \mathrm{CD}^{-} 6^{-}$ macrophages alone may not be able to influence all the other maladaptive processes after TBI. There are conflicting reports of whether adoptive transfer of ex vivo induced M2 macrophages can promote healing after CNS injury $[38,106]$. The M2 phenotype can be further divided into a, b, and c subtypes [107]. M2a phenotypes are promoted by IL- 4 and IL-13 and promote tissue repair and stimulate growth. This is the phenotype we likely enriched and explains the large increase in $\mathrm{CD}^{2} 6^{+}$macrophages as well. M2b macrophages can be proliferative and secrete a mixture of inflammatory and anti-inflammatory cytokines. M2c phenotypes are induced by IL-10 and can promote fibrosis, but are also known to be deactivated. In spinal cord injury, the phenotypes follow each other sequentially with an M2a peak 1-3 dpi and M2b or M2c peak 4-6 dpi. We do not know whether this holds true in TBI; only that M2-like macrophages may peak around day $5[8,9]$ or even day 7 [108] but are quickly overpowered by M1 phenotypes [7]. We also do not know whether the endogenous pattern of sequential phenotypes is ideal for regeneration in neural organs. Getting the right subtype of macrophage, at the right time, may be critical to reducing inflammation and promoting homeostasis. In these subtypes, there might be an explanation for why delivering IL-4 or IL-10 alone has been ineffective. Perhaps, by appropriately timing each cytokine in a concerted approach, M2-like macrophages could proliferate early to overpower maladaptive processes, and then be deactivated as desired. Additionally, we did not scrutinize microglia in our flow cytometry studies as they do not reliably express CD206 [109]. Distinguishing macrophages from microglia is not trivial but IL-4 is known to have favorable effects on both [61, $110,111]$. However, as M1 and M2 phenotypes concurrently exist on microglia, they may need to be assessed for a favorable phenotype after an IL-4 MSC intervention [15]. To obtain some clues to how IL-4 MSCs were affecting microglia, we compared our transcriptomic signatures against a published microglia transcriptomic 
database (S. Table 4). Even with the caveat that our transcripts were obtained from total brain tissue and not isolated microglia, we saw over-representation of microglial "clusters" that suggest a signature of microglia responding to IL-4 only in the IL-4 MSC group (S. Table 4). However, alongside this, all injury groups had clusters suggesting similarities to IFN and LPS stimulation of microglia as well. Therefore, like the macrophages, microglia appear to be receiving numerous signals and IL-4 alone is not enough to adequately modulate the biology. Future studies could isolate macrophages and microglia and perform more precise transcriptomic analyses to further scrutinize the range of phenotypes these cells can possess.

These studies demonstrate a method to augment MSCs with synthetic IL-4 mRNA and a modified closed head injury model of TBI with a behavioral deficit, and also explore the effect of acute IL-4 and M2-like macrophage enrichment after TBI. By inducing MSCs to produce IL-4, we also circumvent the challenges of recombinant protein delivery. Moving forward, it will be useful to explore the effects of dosing, dual-cytokine enrichment, and intervening systemically. Lower doses of either IL-4 mRNA or IL-4 MSCs may promote better healing; although a greater dose may be required for intravenous administration. A few dual-cytokine strategies could include timed deliveries of IL-4 MSCs and IL-10 MSCs, a single delivery of MSCs transfected with IL-4 mRNA (rapid expression) and IL-10 DNA (delayed expression), or intravenous IL-10 bolus a few days after IL-4 MSCs. Additionally, alternate stem cells could be transfected with synthetic IL-4 mRNA, such as MAPCs and umbilical cord cells which may be more effective in TBI than MSCs [43, 47]. Alternatively, MSC exosomes possess benefits over MSCs themselves and could be augmented through transfection [112, 113]. These approaches may also have merit outside TBI, as IL-4 MSCs may facilitate healing in any wounds or environments where proliferation is desired, e.g., in cavities left from disease, or to facilitate tissue integration with implanted biomaterials [22].

Beyond alternative therapeutic approaches, the question remains of how to best modulate inflammation to improve outcomes in TBI. The answer requires a deeper understanding of the roles of macrophage and microglia sub-phenotypes and their interaction with native cells in a TBI environment. Importantly, we need to develop a better understanding of what cytokines and cells are required and when should they be enriched after TBI. As our transcriptomic data suggested that infection and injury have similar pathway enrichment, models of alternate inflammatory stimuli, in alternate organisms, or even an evolution-centered inquiry may provide insight into the maladaptive immunological response seen after TBI [114].

\section{Conclusions}

MSCs that transiently express IL-4 can robustly polarize macrophages toward an M2-like phenotype for at least 1 week after TBI. While this induces cytokine- and genelevel changes, it alone is not enough to significantly impact histological or functional outcomes. Transcriptomic studies reveal persistent inflammatory pathways and an absence of neuroregeneration. However, the question of whether this is due to insufficient or excessive levels of IL-4 or M2-like macrophages, a lack of sensitivity in histological and behavioral measures, our mouse model of TBI, or the ineffectiveness of a single cytokine or cell phenotype in a complex environment, remains.

\section{Supplementary information}

Supplementary information accompanies this paper at https://doi.org/10. 1186/s12974-020-01860-y.

\begin{abstract}
Additional file 1: Figure S1. MSC cell-surface markers. (A) MSCs (Cyagen) used in this study expressed CD29, CD44, and Sca-1 while remaining negative for CD31 and CD45 in at least two late passage numbers.

Additional file 2: Figure S2. Macrophage and MSC control experiments. (A) Flow gating strategy of J774A.1 macrophages prior to individual antibody gates. (B) Fluorescence-minus-one plots to determine antibody gates. (C) Flow plots demonstrating CD163 expression after treatment with differently conditioned media. (D) Brightfield images of macrophages grown in their recommended media (Dulbecco's Modified Eagle Media, DMEM) or MSC growth media. Each image is $1 / 16^{\text {th }}$ of total image analyzed per well. (E) Growth curves of macrophages in each of the media estimated by coverage of the well, calculated from automated imaging scripts. (F) Mean fluorescence intensity of CD206 expression on macrophages after treatment with either $100 \mathrm{ng} / \mathrm{mL}$ recombinant $\mathrm{IL}-4$ ( $\mathrm{rlL}-4)$, conditioned media from MSCs pre-stimulated with $100 \mathrm{ng} / \mathrm{mL} \mathrm{rlL}$ 4 , or conditioned media from naïve MSCs. Mean \pm SD, one- or two-way ANOVA; ${ }^{* * *} p<0.0001$.
\end{abstract}

Additional file 3: Figure S3. Macrophage polarization after closed head injury and treatment alterations through flow cytometry. (A) Pseudocolor flow plots demonstrating gating based on CD45 inensity to identify blood-derived leukocytes in the brain, CD45 ${ }^{\text {high }}$. (B) Flow histograms of FMO controls to assist in gating. (C) - (F) Macrophage analysis 1 week after injury, 2 days after treatment with IL-4 MSCs producing greater IL-4; $n=5, N=10$. (C) Number of total macrophages in the ipsilateral hemisphere for each treatment group. (D) Percentage of all macrophages that possess M2-like phenotype. (E) Number of macrophages with M2-like phenotype. (F) Ratio of M2 to M2-like macrophages. (G) - (I) Macrophage analysis 1 week after injury, 2 days after treatment delivered into the left hippocampus or left lateral ventricle; $n=5, N=10$. (G) Total number of macrophages in the ipsilateral and contralateral hemispheres after either delivery modality. (H) Percent of total macrophages that possess an M2-like phenotype. (I) Number of M2-like macrophages in either hemisphere. Mean \pm SD, Student's t-tests or two-way ANOVAs with posthoc Tukey's; ${ }^{*} p<0.05$, ${ }^{* *} p<0.01$, ${ }^{* * *} p<0.001$, ${ }^{* * *} p<0.0001$.

Additional file 4: Figure S4. (A) - (B) Cytokine analysis of ex vivo injured and treated brain tissue at 1 week after injury. Sham mice or injured mice with day 5 treatment of either PBS, MSCs, or IL-4 MSCs; $n=$ $5, N=20$. (A) Amount of inflammatory cytokine normalized to total protein per hemisphere (Interleukin 2, IL-2; Interferon- $\gamma$, IFNy; and Tumor Necrosis Factor a, TNFa). (B) Amount of anti-inflammatory cytokine normalized to total protein per hemisphere (Interleukin 10, IL-10; Interleukin-13, IL-13; Interleukin-5, IL-5). Graphs display mean \pm SD; one- 
way ANOVA carried out for each cytokine and hemisphere, with Bonferroni-Sidak correction for repeated testing. (C) Gene analysis at 1 week or 3 weeks after injury and day 5 treatment with either PBS, MSCs, or IL-4 MSCs $(n=5)$ and sham mice as biological controls $(n=10) ; \mathrm{N}=$ 40. Heatmap of 26 genes demonstrating up- (red) or down- (blue) regulation of genes based on $\Delta \Delta C T$ values. Boxes with an asterisk $\left(^{*}\right)$ had a corrected $p$-value $<0.05$ as reported by the analysis tool.

Additional file 5: Figure S5. Representative immunohistochemical images of injured and treated brains at 1 and 3 weeks after injury. (A) Section from a sham group brain stained for DAPI, GFAP, and CD68. (B) Section from a sham group stained for DAPI and Fluoro-Jade C. (C)-(E) Representative sections from each injury/treatment group (columns) and timepoint (rows). (C) Stained for DAPI and GFAP. (D) Stained for DAPI and CD68. (E) Stained for DAPI and Fluoro-Jade C.

Additional file 6: Table S1. Fold-changes of significantly altered genes in RT-PCR analysis

Additional file 7: Table S2. Summary and raw data from overrepresentation analysis (ORA)

Additional file 8: Table S3. Summarized raw data from gene set enrichment analysis (GSEA)

Additional file 9: Table S4. Summarized microglia-related overrepresentation analysis (ORA)

\section{Abbreviations}

MSC: Mesenchymal stromal cell; IL-4: Interleukin-4; IL-4 MSC: Interleukin-4 expressing mesenchymal stromal cell; TBI: Traumatic brain injury; WMI: White matter integrity; CHI: Closed head injury; RT: Righting time; NSS: Neurological Severity Score; BBB: Blood-brain barrier; GFP: Green fluorescent protein; IFN: Interferon; LPS: Lipopolysaccharide; PBS: Phosphate-buffered saline: RNA: Ribonucleic acid; mRNA: Messenger ribonucleic acid; RNA-seq: RNA sequencing; MFI: Mean fluorescence intensity; GFAP: Glial fibrillary acidic protein; DAPI: 4',6-Diamidino-2-phenylindole; IHC: Immunohistochemistry; MRI: Magnetic resonance imaging; dpi: Day(s) post-injury; wpi: Week(s) postinjury

\section{Acknowledgements}

We are grateful for the invaluable help of Jared Beyersdorf and Jonathan Kirschman of the Santangelo Lab at the Georgia Institute of Technology for synthesizing IL-4 mRNA in vitro; G. Allan Johnson, Gary Cofer, James J. Cook, and the Center for In Vivo Microscopy at Duke University for magnetic resonance imaging and mapping; and Nicholas Devos, David Corcoran, Heather Hemric, and the Duke Center for Genomic and Computational Biology for RNA and transcriptomic analysis.

\section{Authors' contributions}

SFE, PJS, and RVB designed the studies. SFE and SRK carried out in vivo studies. SFE, NB, and CA carried out in vitro studies. SFE, JGL, and MC analyzed transcriptomic data. PMT, DV, and PJS designed and synthesized mRNA. SFE, SRK, and HW developed the brain injury model. SFE, JGL, MC, $H W$, and RVB wrote or edited the manuscript. All authors approved the final manuscript.

\section{Funding}

This work was supported by the National Institute of Neurological Disorders and Stroke (NINDS), of the National Institutes of Health (NIH), R01NS07973905.

\section{Availability of data and materials}

The datasets generated during the current study are available in the GEO repository, https://www.ncbi.nlm.nih.gov/geo/query/acc.cgi?acc=GSE144193. Additional datasets analyzed during the study are included in supplementary materials with this published article.

Ethics approval and consent to participate

All animal studies were approved by Duke University's Institutional Animal Care and Use Committee

\section{Consent for publication}

Not applicable

\section{Competing interests}

The authors declare that they have no competing interests

\section{Author details}

'Department of Biomedical Engineering, Duke University, Durham, NC, USA ${ }^{2}$ Department of Biomedical Engineering, Georgia Institute of Technology, Atlanta, GA, USA. ${ }^{3}$ Department of Neurology, Duke University, Durham, NC, USA.

Received: 24 February 2020 Accepted: 29 May 2020

Published online: 20 June 2020

\section{References}

1. CDC. Rates of TBI-related emergency department visits, hospitalizations, and deaths - United States, 2001-2010 | Concussion | Traumatic Brain Injury | CDC Injury Center. 2016. p. 1.

2. Faul $\mathrm{M}$, Coronado V. Epidemiology of traumatic brain injury. In: Handbook of clinical neurology. 2015. p. 3-13.

3. CDC Injury Center. Severe TBI | Concussion | Traumatic brain injury | CDC Injury Center. 2016;https://www.cdc.gov/traumaticbraininjury/severe.ht.

4. O'Neil ME, Carlson K, Storzbach D, Brenner L, Freeman M, Quiñones A, et al. Complications of mild traumatic brain injury in veterans and military personnel: a systematic review. Department of Veterans Affairs (US); 2013.

5. Maas Al, Stocchetti N, Bullock R. Moderate and severe traumatic brain injury in adults. Vol. 7, The Lancet Neurology. 2008. p. 728-41.

6. Corps KN, Roth TL, McGavern DB. Inflammation and neuroprotection in traumatic brain injury. JAMA Neurol. 2015;72(3):355-62.

7. Kumar A, Alvarez-Croda DM, Stoica BA, Faden Al, Loane DJ. Microglial/ macrophage polarization dynamics following traumatic brain injury. J Neurotrauma. 2016 Oct 1;33(19):1732-50.

8. Turtzo LC, Lescher J, Janes L, Dean DD, Budde MD, Frank JA. Macrophagic and microglial responses after focal traumatic brain injury in the female rat. J Neuroinflammation. 2014:11(1):82.

9. Wang G, Zhang J, Hu X, Zhang L, Mao L, Jiang X, et al. Microglia/ macrophage polarization dynamics in white matter after traumatic brain injury. J Cereb Blood Flow Metab. 2013 Dec;33(12):1864-74.

10. Kigerl KA, Gensel JC, Ankeny DP, Alexander JK, Donnelly DJ, Popovich PG. Identification of two distinct macrophage subsets with divergent effects causing either neurotoxicity or regeneration in the injured mouse spinal cord. J Neurosci. 2009;29(43):13435-44.

11. Thawer SG, Mawhinney L, Chadwick K, De Chickera SN, Weaver LC, Brown $A$, et al. Temporal changes in monocyte and macrophage subsets and microglial macrophages following spinal cord injury in the lys-egfp-ki mouse model. J Neuroimmunol. 2013;261(1-2):7-20.

12. Oosterhof N, Boddeke E, van Ham TJ. Immune cell dynamics in the CNS learning from the zebrafish. Vol. 63, GLIA. 2015. p. 719-35.

13. Ghosh S, Hui SP. Regeneration of zebrafish CNS: Adult neurogenesis. Vol. 2016, Neural Plasticity. Hindawi Publishing Corporation; 2016. p. 1-21.

14. Gordon S. Alternative activation of macrophages. Vol. 3, Nature Reviews Immunology. 2003. p. 23-35.

15. Morganti JM, Riparip L-K, Rosi S. Call Off the Dog(ma): M1/M2 Polarization Is concurrent following traumatic brain injury. PLoS One. 2016;11(1):e0148001.

16. Enam SF, Krieger JR, Saxena T, Watts BE, Olingy CE, Botchwey EA, et al. Enrichment of endogenous fractalkine and anti-inflammatory cells via aptamer-functionalized hydrogels. Biomaterials. 2017 Oct;142:52-61.

17. Brown BN, Sicari BM, Badylak SF. Rethinking regenerative medicine: a macrophage-centered approach. Vol. 5, Frontiers in Immunology. Frontiers; 2014. p. 510.

18. Italiani P, Boraschi D. From monocytes to M1/M2 macrophages: Phenotypical vs. functional differentiation. Vol. 5, Frontiers in immunology. Frontiers Research Foundation; 2014. p. 514.

19. Shechter R, Miller O, Yovel G, Rosenzweig N, London A, Ruckh J, et al. Recruitment of beneficial M2 macrophages to injured spinal cord is orchestrated by remote brain choroid plexus. Immunity. 2013;38(3):555-69.

20. Shechter R, London A, Varol C, Raposo C, Cusimano M, Yovel G, et al. Infiltrating blood-derived macrophages are vital cells playing an antiinflammatory role in recovery from spinal cord injury in mice. PLoS Med. 2009;6(7):e1000113.

21. Blomster LV, Brennan FH, Lao HW, Harle DW, Harvey AR, Ruitenberg MJ. Mobilisation of the splenic monocyte reservoir and peripheral CX3CR1 
deficiency adversely affects recovery from spinal cord injury. Exp Neurol. 2013;247:226-40.

22. Mokarram N, Merchant A, Mukhatyar V, Patel G, Bellamkonda RV. Effect of modulating macrophage phenotype on peripheral nerve repair. Biomaterials. 2012;33(34):8793-801.

23. Mokarram N, Bellamkonda R V. A perspective on immunomodulation and tissue repair. In: Annals of Biomedical Engineering. 2014. p. 338-51.

24. Hu X, Leak RK, Shi Y, Suenaga J, Gao Y, Zheng P, et al. Microglial and macrophage polarization - new prospects for brain repair. Vol. 11, Nature Reviews Neurology. 2015. p. 56-64.

25. Loane DJ, Kumar A. Microglia in the TBI brain: the good, the bad, and the dysregulated. Vol. 275, Experimental Neurology. 2016. p. 316-27.

26. Morganti JM, Jopson TD, Liu S, Riparip L-K, Guandique CK, Gupta N, et al. CCR2 antagonism alters brain macrophage polarization and ameliorates cognitive dysfunction induced by traumatic brain injury. J Neurosci. 2015.

27. Febinger HY, Thomasy HE, Pavlova MN, Ringgold KM, Barf PR, George AM, et al. Time-dependent effects of CX3CR1 in a mouse model of mild traumatic brain injury. J Neuroinflammation. 2015;12(1):154.

28. Basrai HS, Christie KJ, Turbic A, Bye N, Turnley AM. Suppressor of cytokine signaling-2 (SOCS2) regulates the microglial response and improves functional outcome after traumatic brain injury in mice. PLoS One. 2016;11:4.

29. Wang G, Jiang X, Pu H, Zhang W, An C, Hu X, et al. Scriptaid, a novel histone deacetylase inhibitor, protects against traumatic brain injury via modulation of PTEN and AKT pathway: scriptaid protects against TBI via AKT. Neurotherapeutics. 2013;10(1):124-42.

30. Gao J, Grill RJ, Dunn TJ, Bedi S, Labastida JA, Hetz RA, et al. Human neural stem cell transplantation-mediated alteration of microglial/macrophage phenotypes after traumatic brain injury. Cell Transplant. 2016;25(10):186377.

31. Xu X, Gao W, Cheng S, Yin D, Li F, Wu Y, et al. Anti-inflammatory and immunomodulatory mechanisms of atorvastatin in a murine model of traumatic brain injury. J Neuroinflammation. 2017;14(1):167.

32. Desai A, Park T, Barnes J, Kevala K, Chen H, Kim HY. Reduced acute neuroinflammation and improved functional recovery after traumatic brain injury by a-linolenic acid supplementation in mice. J Neuroinflammation. 2016;13:1.

33. Chen X, Chen C, Fan S, Wu S, Yang F, Fang Z, et al. Omega-3 polyunsaturated fatty acid attenuates the inflammatory response by modulating microglia polarization through SIRT1-mediated deacetylation of the HMGB1/NF-KB pathway following experimental traumatic brain injury. J Neuroinflammation. 2018;15:1.

34. Song G, Yang R, Zhang Q, Chen L, Huang D, Zeng J, et al. TGF- $\beta$ Secretion by $\mathrm{M} 2$ macrophages induces glial scar formation by activating astrocytes in vitro. J Mol Neurosci. 2019 Jul 20;69(2):324-32.

35. Lv J, Zeng Y, Qian Y, Dong J, Zhang Z, Zhang J. MicroRNA let-7c-5p improves neurological outcomes in a murine model of traumatic brain injury by suppressing neuroinflammation and regulating microglial activation. Brain Res. 2018:1685:91-104.

36. Yang Y, Ye Y, Kong C, Su X, Zhang X, Bai W, et al. MiR-124 enriched exosomes promoted the M2 polarization of microglia and enhanced hippocampus neurogenesis after traumatic brain injury by inhibiting TLR4 pathway. Neurochem Res. 2019;44(4):811-28

37. Yao X, Liu S, Ding W, Yue P, Jiang $Q$, Zhao M, et al. TLR4 signal ablation attenuated neurological deficits by regulating microglial M1/M2 phenotype after traumatic brain injury in mice. J Neuroimmunol. 2017;310:38-45.

38. Ma SF, Chen YJ, Zhang JX, Shen L, Wang R, Zhou JS, et al. Adoptive transfer of M2 macrophages promotes locomotor recovery in adult rats after spinal cord injury. Brain Behav Immun. 2015;45:157-70.

39. Loane DJ, Deighan BF, Clarke RM, Griffin RJ, Lynch AM, Lynch MA. Interleukin-4 mediates the neuroprotective effects of rosiglitazone in the aged brain. Neurobiol Aging. 2009;30(6):920-31.

40. Braun M, Khan ZT, Khan MB, Kumar M, Ward A, Achyut BR, et al. Selective activation of cannabinoid receptor-2 reduces neuroinflammation after traumatic brain injury via alternative macrophage polarization. Brain Behav Immun. 2018:68:224-37.

41. Wang G, Shi Y, Jiang X, Leak RK, Hu X, Wu Y, et al. HDAC inhibition prevents white matter injury by modulating microglia/macrophage polarization through the GSK3ß/PTEN/Akt axis. Proc Natl Acad Sci. 2015 Mar 3;112(9): 2853-8
42. Gennai S, Monsel A, Hao Q, Liu J, Gudapati V, Barbier EL, et al. Cell-based therapy for traumatic brain injury. Vol. 115, British Journal of Anaesthesia. Oxford University Press; 2015. p. 203-12.

43. Cox CS. Cellular therapy for traumatic neurological injury. Vol. 83, Pediatric Research. Nature Publishing Group; 2018. p. 325-32.

44. Harting MT, Baumgartner JE, Worth LL, Ewing-Cobbs L, Gee AP, Day M-C, et al. Cell therapies for traumatic brain injury. Neurosurg Focus. 2008 Mar; 24(3-4):E18.

45. Zanier ER, Pischiutta F, Riganti L, Marchesi F, Turola E, Fumagalli S, et al. Bone marrow mesenchymal stromal cells drive protective M2 microglia polarization after brain trauma. Neurotherapeutics. 2014 Jul 26;11(3):679-95.

46. Zanier ER, Montinaro M, Vigano M, Villa P, Fumagalli S, Pischiutta F, et al. Human umbilical cord blood mesenchymal stem cells protect mice brain after trauma. Crit Care Med. 2011;39(11):2501-10.

47. Sun JM, Kurtzberg J. Cord blood for brain injury. Vol. 17, Cytotherapy. 2015. p. $775-85$.

48. Park CW, Kim KS, Bae S, Son HK, Myung PK, Hong HJ, et al. Cytokine secretion profiling of human mesenchymal stem cells by antibody array. Int J Stem Cells. 2009;2(1):59-68.

49. Kyurkchiev D. Secretion of immunoregulatory cytokines by mesenchymal stem cells. World J Stem Cells. 2014;6(5):552.

50. Levy O, Zhao W, Mortensen L, LeBlanc S, Tsang K, Fu M, et al. mRNAengineered mesenchymal stem cells for targeted delivery of interleukin-10 to sites of inflammation. Blood. 2013 Oct 3;122(14):e23-32.

51. Teixeira FG, Carvalho MM, Sousa N, Salgado AJ. Mesenchymal stem cells secretome: a new paradigm for central nervous system regeneration? Vol. 70, Cellular and Molecular Life Sciences. 2013. p. 3871-82.

52. Peruzzaro ST, Andrews MMM, Al-Gharaibeh A, Pupiec O, Resk M, Story D, et al. Transplantation of mesenchymal stem cells genetically engineered to overexpress interleukin-10 promotes alternative inflammatory response in rat model of traumatic brain injury 11 Medical and Health Sciences 1109 Neurosciences. J Neuroinflammation. 2019;16(1):2.

53. Lin T, Pajarinen J, Kohno Y, Maruyama M, Romero-Lopez M, Huang J-F, et al. Transplanted interleukin-4--secreting mesenchymal stromal cells show extended survival and increased bone mineral density in the murine femur. Cytotherapy. 2018 Aug 1;20(8):1028-36.

54. Payne NL, Dantanarayana A, Sun G, Moussa L, Caine S, McDonald C, et al. Early intervention with gene-modified mesenchymal stem cells overexpressing interleukin-4 enhances anti-inflammatory responses and functional recovery in experimental autoimmune demyelination. Cell Adhes Migr. 2012;6(3):179-89.

55. Liu X, Liu J, Zhao S, Zhang H, Cai W, Cai M, et al. Interleukin-4 Is Essential for microglia/macrophage M2 polarization and long-term recovery after cerebral ischemia. Stroke. 2016 Feb 1;47(2):498-504.

56. Zhao X, Wang H, Sun G, Zhang J, Edwards NJ, Aronowski J. Neuronal interleukin- 4 as a modulator of microglial pathways and ischemic brain damage. J Neurosci. 2015 Aug 12;35(32):11281-91.

57. Francos-Quijorna I, Amo-Aparicio J, Martinez-Muriana A, López-Vales R. IL-4 drives microglia and macrophages toward a phenotype conducive for tissue repair and functional recovery after spinal cord injury. Glia. 2016 Dec 1;64(12):2079-92.

58. Lee SI, Jeong SR, Kang YM, Han DH, Jin BK, Namgung U, et al. Endogenous expression of interleukin- 4 regulates macrophage activation and confines cavity formation after traumatic spinal cord injury. J Neurosci Res. 2010; 88(11):2409-19.

59. Lima R, Monteiro S, Lopes JP, Barradas P, Vasconcelos NL, Gomes ED, et al. Systemic interleukin- 4 administration after spinal cord injury modulates inflammation and promotes neuroprotection. Pharmaceuticals. 2017;10:4.

60. Mokarram N, Dymanusb K, Srinivasan A, Lyon JG, Tiptonb J, Chu J, et al. Immunoengineering nerve repair. Proc Natl Acad Sci U S A. 2017;114(26): E5077-84.

61. Gadani SP, Cronk JC, Norris GT, Kipnis J. IL-4 in the brain: a cytokine to remember. J Immunol. 2012 Nov 1;189(9):4213-9.

62. Frugier T, Morganti-Kossmann MC, O'Reilly D, McLean CA. In situ detection of inflammatory mediators in post mortem human brain tissue after traumatic injury. J Neurotrauma. 2010;27(3):497-507.

63. Takamiya M, Fujita S, Saigusa K, Aoki Y. Simultaneous detections of 27 cytokines during cerebral wound healing by multiplexed bead-based immunoassay for wound age estimation. J Neurotrauma. 2007;24(12):183344. 
64. Dalgard CL, Cole JT, Kean WS, Lucky JJ, Sukumar G, McMullen DC, et al. The cytokine temporal profile in rat cortex after controlled cortical impact. Front Mol Neurosci. 2012;(JANUARY 2012).

65. Chen J. Interleukin-4 as a novel therapy for traumatic brain injury (1101BX003377-01) [Internet]. 2016 [cited 2019 Feb 23]. Available from: http://grantome.com/grant/NIH/I01-BX003377-01.

66. Hei Whong, Almansoori AA, Sung MA, Ju KW, Seo N, Lee SH, et al. Adenovirus vector-mediated ex vivo gene transfer of brain-derived neurotrophic factor (BDNF) tohuman umbilical cord blood-derived mesenchymal stem cells (UCB-MSCs) promotescrush-injured rat sciatic nerve regeneration. Neurosci Lett. 2017 Mar 16;643:111-120.

67. Shi X, Bai Y, Zhang G, Liu Y, Xiao H, Liu X, et al. Effects of over-expression of SOD2 in bone marrow-derived mesenchymal stem cells on traumatic brain injury. Cell Tissue Res. 2018 Apr 30;372(1):67-75.

68. Hamzei Taj S, Le Blon D, Hoornaert C, Daans J, Quarta A, Praet J, et al. Targeted intracerebral delivery of the anti-inflammatory cytokine IL13 promotes alternative activation of both microglia and macrophages after stroke. J Neuroinflammation. 2018;15(1):174.

69. Dooley D, Lemmens E, Vangansewinkel T, Le Blon D, Hoornaert C, Ponsaerts $P$, et al. Cell-based delivery of interleukin-13 directs alternative activation of macrophages resulting in improved functional outcome after spinal cord injury. Stem Cell Reports. 2016;7(6):1099-115

70. Ali I, Aertgeerts S, Le Blon D, Bertoglio D, Hoornaert C, Ponsaerts P, et al. Intracerebral delivery of the M2 polarizing cytokine interleukin 13 using mesenchymal stem cell implants in a model of temporal lobe epilepsy in mice. Epilepsia. 2017;58(6):1063-72.

71. Luzina IG, Keegan AD, Heller NM, Rook GAW, Shea-Donohue T, Atamas SP. Regulation of inflammation by interleukin-4: a review of "alternatives". J Leukoc Biol. 2012;92(4):753-64.

72. Fernández-Klett F, Priller J. The fibrotic scar in neurological disorders. In: Brain pathology. Blackwell Publishing Ltd; 2014. p. 404-13.

73. Werle M, Bernkop-Schnürch A. Strategies to improve plasma half life time of peptide and protein drugs. Vol. 30, Amino Acids. 2006. p. 351-67.

74. Vugmeyster Y, Xu X, Theil F-P, Khawli LA, Leach MW. Pharmacokinetics and toxicology of therapeutic proteins: advances and challenges. World J Biol Chem. 2012 Apr 26:3(4):73-92.

75. Solá RJ, Griebenow K. Glycosylation of therapeutic proteins: an effective strategy to optimize efficacy. Vol. 24, BioDrugs. NIH Public Access; 2010. p. 9-21.

76. Schellekens $\mathrm{H}$. Factors influencing the immunogenicity of therapeutic proteins. Nephrol Dial Transplant. 2005 Jun 1;20(suppl_6):vi3-9.

77. Schellekens H. Immunogenicity of therapeutic proteins: clinical implications and future prospects. Clin Ther. 2002;24(11):1720-40.

78. Conlon PJ, Tyler S, Grabstein KH, Morrissey P. Interleukin-4 (B-cell stimulatory factor-1) augments the in vivo generation of cytotoxic cells in immunosuppressed animals. Biotechnol Ther. 1989;1(1):31-41.

79. Xu Y, Huang L, Kirschman JL, Vanover DA, Tiwari PM, Santangelo PJ, et al. Exploitation of synthetic mRNA to drive immune effector cell recruitment and functional reprogramming in vivo. J Immunol. 2019 Jan 15;202(2):608-17.

80. Webster SJ, Van Eldik LJ, Martin Watterson D, Bachstetter AD. Closed head injury in an age-related alzheimer mouse model leads to an altered neuroinflammatory response and persistent cognitive impairment. J Neurosci. 2015;35(16):6554-69.

81. Grin'kina NM, Li Y, Haber M, Sangobowale M, Nikulina E, Le'pre C, et al. Righting reflex predicts long-term histological and behavioral outcomes in a closed head model of traumatic brain injury. PLoS One. 2016;11(9): e0161053.

82. Flierl MA, Stahel PF, Beauchamp KM, Morgan SJ, Smith WR, Shohami E Mouse closed head injury model induced by a weight-drop device. Nat Protoc. 2009 Sep 27:4(9):1328-37.

83. Pösel C, Möller K, Boltze J, Wagner DC, Weise G. Isolation and flow cytometric analysis of immune cells from the ischemic mouse brain. J Vis Exp. 2016;2016:108.

84. Mestdagh $P$, Van Vlierberghe P, De Weer A, Muth D, Westermann F, Speleman F, et al. A novel and universal method for microRNA RT-qPCR data normalization. Genome Biol. 2009 Jun 16;10(6):R64.

85. Frankish A, Diekhans M, Ferreira AM, Johnson R, Jungreis I, Loveland J, et al. GENCODE reference annotation for the human and mouse genomes. Nucleic Acids Res. 2019 Jan 8;47(D1):D766-73.
86. Bray NL, Pimentel H, Melsted P, Pachter L. Near-optimal probabilistic RNAseq quantification. Nat Biotechnol. 2016;34(5):525-7.

87. Pimentel H, Bray NL, Puente S, Melsted P, Pachter L. Differential analysis of RNA-seq incorporating quantification uncertainty. Nat Methods. 2017;14(7): 687-90.

88. Reimand J, Arak T, Adler P, Kolberg L, Reisberg S, Peterson H, et al. g: Profiler-a web server for functional interpretation of gene lists (2016 update). Nucleic Acids Res. 2016;44(W1):W83-9.

89. Subramanian A, Tamayo P, Mootha VK, Mukherjee S, Ebert BL, Gillette MA, et al. Gene set enrichment analysis: a knowledge-based approach for interpreting genome-wide expression profiles. Proc Natl Acad Sci. 2005 Oct 25;102(43):15545-50.

90. Wang N, Anderson RJ, Badea A, Cofer G, Dibb R, Qi Y, et al. Whole mouse brain structural connectomics using magnetic resonance histology. Brain Struct Funct. 2018;223(9):4323-35.

91. Johnson GA, Badea A, Brandenburg J, Cofer G, Fubara B, Liu S, et al. Waxholm Space: an image-based reference for coordinating mouse brain research. Neuroimage. 2010;53(2):365-72.

92. Calabrese E, Badea A, Cofer G, Qi Y, Johnson GA. A Diffusion MRI tractography connectome of the mouse brain and comparison with neuronal tracer data. Cereb Cortex. 2015;25(11):4628-37.

93. Baddoo M, Hill K, Wilkinson R, Gaupp D, Hughes C, Kopen GC, et al. Characterization of mesenchymal stem cells isolated from murine bone marrow by negative selection. J Cell Biochem. 2003;89(6):1235-49.

94. Jenkins SJ, Ruckerl D, Cook PC, Jones LH, Finkelman FD, Van Rooijen N, et al. Local macrophage proliferation, rather than recruitment from the blood, is a signature of T H2 inflammation. Science (80- ). 2011;332(6035):1284-8.

95. Casella G, Garzetti L, Gatta AT, Finardi A, Maiorino C, Ruffini F, et al. IL4 induces IL6-producing M2 macrophages associated to inhibition of neuroinflammation in vitro and in vivo. J Neuroinflammation. 2016;13(1).

96. Kumar RG, Diamond ML, Boles JA, Berger RP, Tisherman SA, Kochanek PM, et al. Acute CSF interleukin-6 trajectories after TBI: associations with neuroinflammation, polytrauma, and outcome. Brain Behav Immun. 2015;45: 253-62.

97. Erta M, Quintana A, Hidalgo J. Interleukin-6, a major cytokine in the central nervous system. Int J Biol Sci. 2012;8(9):1254-66.

98. Yang SH, Gangidine M, Pritts TA, Goodman MD, Lentsch AB. Interleukin 6 mediates neuroinflammation and motor coordination deficits after mild traumatic brain injury and brief hypoxia in mice. Shock. 2013 Dec;40(6):471-5.

99. Haber M, Hutchinson EB, Sadeghi N, Cheng WH, Namjoshi D, Cripton P, et al. Defining an analytic framework to evaluate quantitative MRI markers of traumatic axonal injury: Preliminary results in a mouse closed head injury model. eNeuro. 2017:4:5

100. Yu F, Shukla DK, Armstrong RC, Marion CM, Radomski KL, Selwyn RG, et al. Repetitive model of mild traumatic brain injury produces cortical abnormalities detectable by magnetic resonance diffusion imaging, histopathology, and behavior. J Neurotrauma. 2017;34(7):1364-81.

101. Bodnar CN, Roberts KN, Higgins EK, Bachstetter AD. A systematic review of closed head injury models of mild traumatic brain injury in mice and rats. Vol. 36, Journal of Neurotrauma. 2019. p. 1683-706.

102. Harting MT, Jimenez F, Xue H, Fischer UM, Baumgartner J, Dash PK, et al. Intravenous mesenchymal stem cell therapy for traumatic brain injury: laboratory investigation. J Neurosurg. 2009;110(6):1189-97.

103. Shlosberg D, Benifla M, Kaufer D, Friedman A. Blood-brain barrier breakdown as a therapeutic target in traumatic brain injury. Vol. 6, Nature Reviews Neurology. 2010. p. 393-403.

104. Knoblach SM, Faden Al. Interleukin-10 improves outcome and alters proinflammatory cytokine expression after experimental traumatic brain injury. Exp Neurol. 1998 Sep 1;153(1):143-51.

105. Willing AE, Lixian J, Milliken M, Poulos S, Zigova T, Song S, et al. Intravenous versus intrastriatal cord blood administration in a rodent model of stroke. J Neurosci Res. 2003;73(3):296-307.

106. Desestret V, Riou A, Chauveau F, Cho TH, Devillard E, Marinescu M, et al. In vitro and in vivo models of cerebral ischemia show discrepancy in therapeutic effects of M2 macrophages. PLoS One. 2013;8:6.

107. Gensel JC, Zhang B. Macrophage activation and its role in repair and pathology after spinal cord injury. Brain Res. 2015;1619:1-11.

108. Jin X, Ishii H, Bai Z, Itokazu T, Yamashita T. Temporal changes in cell marker expression and cellular infiltration in a controlled cortical impact model in adult male C57BL/6 mice. PLoS One. 2012:7:7. 
109. Rossi C, Cusimano M, Zambito M, Finardi A, Capotondo A, Garcia-Manteiga $\mathrm{JM}$, et al. Interleukin 4 modulates microglia homeostasis and attenuates the early slowly progressive phase of amyotrophic lateral sclerosis article. Cell Death Dis. 2018;9(2):250

110. Butovsky O, Ziv Y, Schwartz A, Landa G, Talpalar AE, Pluchino S, et al. Microglia activated by IL-4 or IFN- $\gamma$ differentially induce neurogenesis and oligodendrogenesis from adult stem/progenitor cells. Mol Cell Neurosci. 2006:31(1):149-60.

111. Spittau B. Interleukin 4-induced neuroprotection and regulation of microglia activation as a therapeutic approach in the MPTP model of parkinson's disease. Vol. 12, Neural Regeneration Research. 2017. p. 1433-4.

112. Zhang ZG, Buller B, Chopp M. Exosomes - beyond stem cells for restorative therapy in stroke and neurological injury. Vol. 15, Nature Reviews Neurology. 2019. p. 193-203.

113. Zhang Y, Chopp M, Meng Y, Katakowski M, Xin H, Mahmood A, et al. Effect of exosomes derived from multipluripotent mesenchymal stromal cells on functional recovery and neurovascular plasticity in rats after traumatic brain injury. J Neurosurg. 2015;122(4):856-67.

114. Weil ZM, Norman GJ, DeVries AC, Nelson RJ. The injured nervous system: a Darwinian perspective. Vol. 86, Progress in Neurobiology. NIH Public Access; 2008. p. 48-59.

\section{Publisher's Note}

Springer Nature remains neutral with regard to jurisdictional claims in published maps and institutional affiliations.

Ready to submit your research? Choose BMC and benefit from:

- fast, convenient online submission

- thorough peer review by experienced researchers in your field

- rapid publication on acceptance

- support for research data, including large and complex data types

- gold Open Access which fosters wider collaboration and increased citations

- maximum visibility for your research: over $100 \mathrm{M}$ website views per year

At $\mathrm{BMC}$, research is always in progress.

Learn more biomedcentral.com/submissions 